\title{
NONLOCAL CAHN-HILLIARD-NAVIER-STOKES SYSTEMS WITH SHEAR DEPENDENT VISCOSITY
}

\author{
SERGIO FRIGERI*, MAURIZIO GRASSELLI ${ }^{\dagger}$, DALIBOR PRAŽÁK ${ }^{\ddagger}$
}

\begin{abstract}
We consider a diffuse interface model for the phase separation of an incompressible and isothermal non-Newtonian binary fluid mixture in three dimensions. The averaged velocity $\boldsymbol{u}$ is governed by a Navier-Stokes system with a shear dependent viscosity controlled by a power $p>2$. This system is nonlinearly coupled through the Korteweg force with a convective nonlocal Cahn-Hilliard equation for the order parameter $\varphi$, that is, the (relative) concentration difference of the two components. The resulting equations are endowed with the no-slip boundary condition for $\boldsymbol{u}$ and the no-flux boundary condition for the chemical potential $\mu$. The latter variable is the functional derivative of a nonlocal and nonconvex Ginzburg-Landau type functional which accounts for the presence of two phases. We first prove the existence of a weak solution in the case $p \geq 11 / 5$. Then we extend some previous results on time regularity and uniqueness if $p>11 / 5$.
\end{abstract}

\section{InTRODUCTION}

We consider a mixture of incompressible, isothermal and (partially) immiscible binary fluids in a given bounded domain $\Omega \subset \mathbb{R}^{3}$. We suppose that they both have density equal to one and we denote by $\boldsymbol{u}$ their (volume) averaged velocity and by $\varphi$ the (relative) concentration difference. A well-known diffuse interface model (see, e.g., [4, 25, 26]) for the phase separation of the mixture is given by

$$
\begin{aligned}
\partial_{t} \boldsymbol{u}+(\boldsymbol{u} \cdot \nabla) \boldsymbol{u}-\operatorname{div} \mathcal{S}(\varphi, D \boldsymbol{u})+\nabla \pi & =\mu \nabla \varphi+h(t) \\
\operatorname{div} \boldsymbol{u} & =0 \\
\partial_{t} \varphi+(\boldsymbol{u} \cdot \nabla) \varphi & =\Delta \mu \\
\mu & =-\Delta \varphi+F^{\prime}(\varphi)
\end{aligned}
$$

in $\Omega \times(0, T), T>0$. Here the mobility and other constants have been taken equal to one, $F$ is a double well potential (e.g., $F(r)=\left(r^{2}-1\right)^{2}, r \in \mathbb{R}$ ) which accounts for the presence of two components, $\boldsymbol{h}$ is an external force. The stress tensor $\mathcal{S}$, up to the pressure term, depends on the symmetric gradient $D \boldsymbol{u}:=\left(\nabla \boldsymbol{u}+\nabla^{T} \boldsymbol{u}\right) / 2$ of the velocity field $\boldsymbol{u}$ and, possibly, on $\varphi$, through a suitable constitutive law. If, for instance, we have

$$
\mathcal{S}(\varphi, D \boldsymbol{u})=\nu(\varphi) D \boldsymbol{u},
$$

$\nu$ being a given strictly positive function, then we are in presence of a Newtonian mixture. The corresponding system (1.1)-(1.4) is called Cahn-Hilliard-Navier-Stokes system (see,

Date: July 15, 2017.

2010 Mathematics Subject Classification. 35Q35,76A05,76D03,76T10.

Key words and phrases. Incompressible viscous fluids, shear dependent viscosity, binary mixtures, diffuse interfaces, nonlocal Cahn-Hilliard equations, uniqueness, regularity. 
e.g., $[1,8,9,20,30,34,36])$. When the mixture has non-Newtonian features, then the stress tensor itself depends on some power of $|D \boldsymbol{u}|$. A typical example is given by

$$
\mathcal{S}(\varphi, D \boldsymbol{u})=\left(\nu_{1}(\varphi)+\nu_{2}(\varphi)|D \boldsymbol{u}|^{p-2}\right) D \boldsymbol{u}
$$

where $\nu_{i}, i=1,2$, are strictly positive functions and $p>1$. Concerning the single nonNewtonian fluids see, for instance, [32] for the physical background, and [31] for the basic mathematical theory; cf. also [10, 13] and its references for more advanced development. More recently, system (1.1)-(1.4) has also been investigated in a number of contributions (see $[2,7,27,23,24]$ ). In those papers, the chemical potential $\mu$ (see (1.1)) is the functional derivative of the Ginzburg-Landau type functional

$$
\mathcal{F}(\varphi)=\int_{\Omega}\left(\frac{|\nabla \varphi(x)|^{2}}{2}+F(\varphi(x))\right) d x
$$

However, this is a phenomenological assumption and a more rigorous approach shows that the functional should be nonlocal (see [21, 22]). For instance, following [5], we can take

$$
\mathcal{E}(\varphi)=\frac{1}{4} \int_{\Omega \times \Omega} J(x-y)|\varphi(x)-\varphi(y)|^{2} d x d y+\int_{\Omega} F(\varphi(x)) d x .
$$

Here $J: \mathbb{R} \rightarrow \mathbb{R}$ is a sufficiently smooth interaction kernel such that $J(x)=J(-x)$. With this choice the chemical potential becomes

$$
\mu=a \varphi-J * \varphi+F^{\prime}(\varphi)
$$

where

$$
a(x):=\int_{\Omega} J(x-y) d y, \quad(J * \varphi)(x)=\int_{\Omega} J(x-y) \varphi(y) d y .
$$

Therefore we have the following nonlocal system in $\Omega \times(0, T)$

$$
\begin{aligned}
\partial_{t} \boldsymbol{u}+(\boldsymbol{u} \cdot \nabla) \boldsymbol{u}-\operatorname{div} \mathcal{S}(\varphi, D \boldsymbol{u})+\nabla \pi & =\mu \nabla \varphi+\boldsymbol{h}(t) \\
\operatorname{div} \boldsymbol{u} & =0 \\
\partial_{t} \varphi+(\boldsymbol{u} \cdot \nabla) \varphi & =\Delta \mu \\
\mu & =a \varphi-J * \varphi+F^{\prime}(\varphi) .
\end{aligned}
$$

In the case (1.5), system (1.9)-(1.12) has been studied first in [11]. Then, under various assumptions and generalizations, in several other papers (see, for instance, $[15,16,17,14$, $18]$ ). Here we want to analyze this system within a reasonably simple (but meaningful) non-Newtonian setting. Namely, we assume that $\mathcal{S}$ only depends on $D \boldsymbol{u}$ with a $(p-1)$ power growth (cf. (1.6) and see Section 2 for all the assumptions). Moreover, we suppose that $F$ is smooth enough with a polynomially controlled growth and satisfies a coercivity condition of order $2 q+2$, for some $q \geq 0$ (see Section 2 for the details). Summing up, here we consider the system

$$
\begin{aligned}
\partial_{t} \boldsymbol{u}+(\boldsymbol{u} \cdot \nabla) \boldsymbol{u}-\operatorname{div} \mathcal{S}(D \boldsymbol{u})+\nabla \pi & =\mu \nabla \varphi+\boldsymbol{h}(t) \\
\operatorname{div} \boldsymbol{u} & =0 \\
\partial_{t} \varphi+(\boldsymbol{u} \cdot \nabla) \varphi & =\Delta \mu \\
\mu & =a \varphi-J * \varphi+F^{\prime}(\varphi)
\end{aligned}
$$


in $\Omega \times(0, T)$, subject to the initial and boundary conditions

$$
\begin{aligned}
& \boldsymbol{u}=\mathbf{0}, \quad \frac{\partial \mu}{\partial n}=0 \quad \text { on } \partial \Omega \times(0, T) \\
& \boldsymbol{u}(0)=\boldsymbol{u}_{0}, \varphi(0)=\varphi_{0} \quad \text { in } \Omega .
\end{aligned}
$$

Our first result is the existence of a global weak solution (see [11] for the case (1.5)). We point out that the basic energy estimate essentially yields (see Section 4)

$\boldsymbol{u} \in L^{\infty}\left(0, T ; L^{2}(\Omega)\right) \cap L^{p}\left(0, T ;\left(W^{1, p}(\Omega)\right)^{3}\right), \quad \varphi \in L^{\infty}\left(0, T ; L^{2 q+2}(\Omega)\right) \cap L^{2}\left(0, T ; W^{1,2}(\Omega)\right)$. If $p \geq 11 / 5$ and $q \geq q_{p}$, where $q_{p}$ is given by (3.13) below, this regularity is enough to prove that $\partial_{t} \boldsymbol{u}$ and $\partial_{t} \varphi$ belong to the respective dual spaces. This fact has the following consequences:

(i) the couple $(\boldsymbol{u}, \mu)$ is an admissible test function for (1.13) and (1.15), respectively;

(ii) any weak solution satisfies the energy identity;

(iii) existence of weak solutions can be shown using the monotone operator theory.

The above restrictions on $p$ and $q$ are not necessary, however, if merely the problem of existence of solutions is considered. For instance, the value $q=0$ is enough to handle the equation for $\varphi$, provided that $p$ is large enough. The equation for $\boldsymbol{u}$ is more delicate; but it is possible to pass to the limit by a subtle modification of Minty's trick, taking a suitable truncation of $\boldsymbol{u}$ as a test function. This so-called Lipschitz truncation method enables one to prove the existence of solutions down to the value $p=6 / 5$, which seems optimal as for even lower values of $p$, the convective term in (1.13) ceases to be integrable (see [13]).

On the other hand, it does not seem possible to further relax the condition $p \geq 11 / 5$ as issues (i) and (ii) are concerned. A fortiori, the uniqueness of weak solutions certainly cannot be shown for smaller $p$ (note that $p=2$ includes the incompressible Navier-Stokes equations as a special case). Resting on the estimates of differences of two solutions, in view of the nonlinear character of the problem, strictly higher values of $p$ and $q$ seem necessary. In particular, as was already observed already for single fluids (see [28]), the class of weak solutions is unique provided that $p \geq 5 / 2$. More precisely, speaking of single fluids, any weak solution with additional regularity $\boldsymbol{u} \in L^{p_{\text {uniq }}}\left(0, T ;\left(W^{1, p}(\Omega)\right)^{3}\right)$ is unique among all the weak solutions, where $p_{\text {uniq }}=2 p /(2 p-3)$. Note that weak solutions have this regularity if $p \geq 5 / 2$.

This further motivates the search for additional regularity of weak solutions. In view of the above observations, a slight improvement of the time regularity would imply their uniqueness. This issue has been solved in a quite satisfactory way in [10]. Here it was shown, roughly speaking, that if $p>11 / 5$ then any weak solution, considered as a timedependent function with values in $\left(W^{1, p}(\Omega)\right)^{3}$, has locally fractional time regularity analogous to the regularity of the external force $\boldsymbol{h}$, considered as a function into the dual space of $\left(W^{1, p}(\Omega)\right)^{3}$. This was made possible by using the class of Nikolskii spaces.

Here we extend the result to (1.15)-(1.18) (cf. [24] for a similar result in the case (1.1)(1.4)). We also improve the result slightly, by showing that under explicit and natural conditions on the initial data, the time regularity holds globally, i.e., up to $t=0$.

This fact has two expected corollaries. First of all, the uniqueness assertion implies that any approximation scheme yields the same weak solution, with all the regularity obtained along the way provided the data are regular enough. In particular, the $L^{\infty}(\Omega \times(0, T))$ bound for $\varphi$ can be devised, using a Alikakos-Moser type scheme. A second general 
consequence is the unique continuation property: if two weak solutions coincide on some nontrivial interval $\left[t_{0}, \tau\right], t_{0} \geq 0$, then they also coincide for all $t>\tau$. This implies, together with the above-mentioned energy identities, that the existence of global and exponential attractors for large times can be proven within the setting of $\ell$-trajectories. We however do not elaborate on this point further, as an otherwise standard machinery is employed (see [23, Section 3], cf. also [7, 24]).

\section{BASIC ASSUMPtiOns, FUNCTION SPACES AND OPERATORS}

Let us begin with the assumptions on $\mathcal{S}, J$ and $F$ which will be used in the sequel. We suppose:

- $\mathcal{S}(\cdot)$ continuously depends on a symmetric tensor $\boldsymbol{e} \in \mathbb{R}^{3 \times 3}$ and satisfies the following conditions

$$
\begin{aligned}
\left(\mathcal{S}\left(\boldsymbol{e}_{1}\right)-\mathcal{S}\left(\boldsymbol{e}_{2}\right)\right):\left(\boldsymbol{e}_{1}-\boldsymbol{e}_{2}\right) & \geq\left\{\begin{array}{l}
c_{1}\left(1+\left|\boldsymbol{e}_{1}\right|+\left|\boldsymbol{e}_{2}\right|\right)^{p-2}\left|\boldsymbol{e}_{1}-\boldsymbol{e}_{2}\right|^{2} \\
c_{2}\left|\boldsymbol{e}_{1}-\boldsymbol{e}_{2}\right|^{2}+c_{2}\left|\boldsymbol{e}_{1}-\boldsymbol{e}_{2}\right|^{p}
\end{array}\right. \\
\left|\mathcal{S}\left(\boldsymbol{e}_{1}\right)-\mathcal{S}\left(\boldsymbol{e}_{2}\right)\right| & \leq c_{3}\left(1+\left|\boldsymbol{e}_{1}\right|+\left|\boldsymbol{e}_{2}\right|\right)^{p-2}\left|\boldsymbol{e}_{1}-\boldsymbol{e}_{2}\right|, \quad \mathcal{S}(\mathbf{0})=\mathbf{0},
\end{aligned}
$$

for all $\boldsymbol{e}_{1}, \boldsymbol{e}_{2} \in \mathbb{R}^{3 \times 3}$, for some $c_{i}>0, i=1,2,3$, and some $p>2$. Here $|\cdot|$ stands for the Euclidean norm of a tensor.

- $J \in W_{l o c}^{1,1}\left(\mathbb{R}^{3}\right)$. Moreover, we set

$$
a^{*}:=\sup _{x \in \Omega} \int_{\Omega}|J(x-y)| d y<\infty, \quad b:=\sup _{x \in \Omega} \int_{\Omega}|\nabla J(x-y)| d y<\infty .
$$

- $F \in C^{2}(\mathbb{R})$ has a polynomially controlled growth

$$
\left|F^{\prime}(s)\right|^{r} \leq c_{4}(|F(s)|+1), \quad r \in(1,2]
$$

for some $c_{4}>0$ and satisfies the coercivity condition:

$$
F^{\prime \prime}(s)+a(x) \geq c_{5} \max \left\{1,|s|^{2 q}\right\}
$$

for all $s \in \mathbb{R}$, almost any $x \in \Omega$, some $c_{5}>0$ and some $q \geq 0$.

Here $a$ is defined by (1.8).

Remark 1. From the mathematical viewpoint assumption (2.3) is satisfied, in particular, if $a \equiv 0$ and $F$ (strictly) convex. This assumption is physically justified and relevant (see [19] for a detailed discussion).

We employ the standard Lebesgue and Sobolev spaces, pertinent to the weak formulation of our problem, namely, setting

$$
\mathcal{V}:=\left\{\boldsymbol{v} \in \mathcal{D}(\Omega)^{3}: \operatorname{div} \boldsymbol{v}=0\right\}
$$

we define

$$
G:=\overline{\mathcal{V}}^{L^{2}\left(\Omega ; \mathbb{R}^{3}\right)}, \quad V_{p}:=\overline{\mathcal{V}}^{W^{1, p}\left(\Omega ; \mathbb{R}^{3}\right)} .
$$

For other Hilbert spaces $X$ the scalar product will be denoted by $(\cdot, \cdot)_{X}$. The notation $\langle\cdot, \cdot\rangle_{Y}$ and $\|\cdot\|_{Y}$ will stand for the duality pairing between a Banach space $Y$ and its dual $Y^{\prime}$, and for the norm of $Y$, respectively. In order to simplify the notation, we indicate the norms $\|\cdot\|_{W^{s, p}(\Omega)}$ and duality pairings $\langle\cdot, \cdot\rangle_{W^{s, p}(\Omega)}$ always as $\|\cdot\|_{W^{s, p}}$ and $\langle\cdot, \cdot\rangle_{W^{s, p}}$, respectively, for all $s \in \mathbb{R}$ and all $p \in[1, \infty]$. 
Moreover, we introduce

$$
H_{(0)}^{s}(\Omega):=\left\{\psi \in H^{s}(\Omega):\langle\psi, 1\rangle_{H^{s}}=0\right\},
$$

for $s \in \mathbb{R}$, and we set

$$
\|u\|_{H^{s}}^{2}=\sum_{j \in \mathbb{N}} \lambda_{j}^{s} c_{j}^{2} \quad c_{j}=\int_{\Omega} u(x) \omega_{j}(x) d x,
$$

where $\left\{\left(\lambda_{j}, \omega_{j}\right)\right\}_{j \in \mathbb{N}}$ are the eigenvalues and eigenfunctions of the weak Laplace operator $B_{N}$ with homogenous Neumann boundary condition, that is, for $f \in H^{1}(\Omega)^{\prime}$ and $u \in$ $H^{1}(\Omega)$ we have

$$
B_{N} u=f \quad \Longleftrightarrow \quad \int_{\Omega} \nabla u \cdot \nabla \phi=\langle f, \phi\rangle_{H^{1}} \quad \forall \phi \in H^{1}(\Omega) .
$$

We recall that $B_{N}$ is an isometry between $H_{(0)}^{1}(\Omega)$ and the space

$$
H_{(0)}^{-1}(\Omega):=\left\{f \in H^{1}(\Omega)^{\prime}:\langle f, 1\rangle_{H^{1}}=0\right\} .
$$

In particular, we have

$$
\left\|B_{N}^{-1} \phi\right\|_{H^{1}} \leq c\|\phi\|_{\left(H^{1}\right)^{\prime}}, \quad\left\|B_{N}^{-1} \phi\right\|_{H^{2}} \leq c\|\phi\| .
$$

Our main focus will be the time regularity of vector-valued function $u:[0, T] \rightarrow X$, where $X$ is some (real) Banach space. The symbol $\frac{d}{d t}$ denotes the weak (distributional) derivative, and $C(\cdot), C^{0, \lambda}(\cdot)$ indicates the usual spaces of continuous and $\lambda$-Hölder continuous functions, respectively. To describe a finer scale of fractional time regularity, we work with the so-called Nikolskii spaces. For $u: I \rightarrow X$, where $I \subset \mathbb{R}$ is an arbitrary time interval, and $h>0$, we set

$$
\begin{aligned}
I_{h} & :=\{t \in I ; t+h \in I\} \\
\tau^{h} u(t) & :=u(t+h), \quad t \in I_{h} \\
d^{h} u(t) & :=u(t+h)-u(t), \quad t \in I_{h} .
\end{aligned}
$$

For $p \in[1, \infty]$ and $s \in(0,1)$, the Nikolskii space $N^{s, p}(I ; X)$ is defined through the norm

$$
\|u\|_{L^{p}(I ; X)}+\sup _{h>0} h^{-s}\left\|d^{h} u\right\|_{L^{p}\left(I_{h} ; X\right)} .
$$

It is not difficult to see that for $s=1$, the above norm is equivalent to $W^{1, p}(I ; X)$. For a general $\sigma=k+s$, where $k \in \mathbb{N}$ and $s \in(0,1)$, one defines $N^{\sigma, p}(I ; X)$ as the space of functions such that $\left(\frac{d}{d t}\right)^{j} u \in L^{p}(I, X)$ for $j=0, \ldots, k$ and, moreover, $\left(\frac{d}{d t}\right)^{k} u \in N^{s, p}(I ; X)$.

Nikolskii spaces are fractional regularity spaces. In particular, we recall the identity $N^{s, p}(I ; X)=B_{\infty}^{s, p}(I ; X)$, where the latter is a Besov space. The corresponding theory is treated in several texts (see, e.g., [3] or [6]). Relatively elementary treatment can be found in [33]. The following embeddings are standard (cf., for instance, [33, Corollary 26 and 33])

$$
\begin{aligned}
& N^{s, p}(I ; X) \hookrightarrow C^{0, \lambda}(I ; X) \quad \text { if } \lambda=s-\frac{1}{p}>0 \\
& N^{s, p}(I ; X) \hookrightarrow L^{q}(I ; X) \quad \text { if } q<\widetilde{p}_{s}, \quad \frac{1}{\widetilde{p}_{s}}:=\frac{1}{p}-s \geq 0 .
\end{aligned}
$$

Nikolskii spaces are not the best choice in view of interpolation or embedding results; note the strict condition on $q$ in (2.8). Their relative advantage lies in the simplicity of their 
definition. Indeed, we will see that it is rather straightforward to obtain estimates of the $N^{s, p}$-norm. The following special interpolation result will be useful (see [10, Lemma 2.3] for a simple proof).

Lemma 1. Let $X \hookrightarrow H$, where $H$ is a Hilbert space and $X$ is separable and dense in $H$. Then

$$
N^{\alpha, p}(I ; X) \cap N^{\beta, p^{\prime}}\left(I ; X^{\prime}\right) \hookrightarrow N^{\frac{\alpha+\beta}{2}, 2}(I ; H)
$$

for any $\alpha, \beta \geq 0$.

Concerning the boundary regularity, we take $\partial \Omega$ of class $C^{2,1}$ for the sake of simplicity. However, a careful look at the approximation scheme used for the existence (see Section 4) shows that a Lipschitz boundary would suffice. This holds true for the time regularity as well.

\section{MAin RESUlts}

Here we introduce the weak formulation of problem (1.15)-(1.18) and we state our main results.

First, let us introduce the following bilinear and trilinear forms

$$
\begin{aligned}
\langle N(\boldsymbol{u}), \mathbf{v}\rangle_{V_{p}} & =\int_{\Omega} \mathcal{S}(D \boldsymbol{u}): D \mathbf{v} d x \\
\left\langle K_{0}(\boldsymbol{u}), \mathbf{v}\right\rangle_{V_{p}} & =\int_{\Omega}(\boldsymbol{u} \otimes \boldsymbol{u}): \nabla \mathbf{v} d x \\
\left\langle K_{1}(\varphi), \mathbf{v}\right\rangle_{V_{p}} & =-\frac{1}{2} \int_{\Omega} \varphi^{2} \nabla a \cdot \mathbf{v} d x+\int_{\Omega}(\nabla J * \varphi) \varphi \cdot \mathbf{v} d x \\
\left\langle K_{2}(\boldsymbol{u}, \varphi), \psi\right\rangle_{H^{1}} & =\int_{\Omega} \varphi \boldsymbol{u} \cdot \nabla \psi d x
\end{aligned}
$$

which are well defined for all $\boldsymbol{u}, \mathbf{v} \in V_{p}$ and for all $\varphi \in L^{2 q+2}(\Omega)$ and $\psi \in H^{1}(\Omega)$, where $p$ and $q$ are chosen as in Theorem 1 below.

Definition 1. A pair $[\boldsymbol{u}, \varphi]$ will be called weak solution to (1.13)-(1.17), if

$$
\begin{aligned}
\boldsymbol{u} & \in L^{\infty}(0, T ; G) \cap L^{p}\left(0, T ; V_{p}\right) \\
\varphi & \in L^{\infty}\left(0, T ; L^{2 q+2}(\Omega)\right) \cap L^{2}\left(0, T ; H^{1}(\Omega)\right) \\
a \varphi-J * \varphi+F^{\prime}(\varphi) & \in L^{2}\left(0, T ; H^{1}(\Omega)\right)
\end{aligned}
$$

and

$$
\begin{aligned}
& \frac{d}{d t} \boldsymbol{u}+N(\boldsymbol{u})=K_{0}(\boldsymbol{u})+K_{1}(\varphi)+\boldsymbol{h}(t) \quad \text { in } V_{p}^{\prime}, \quad \text { a.e. in }(0, T) \\
& \frac{d}{d t} \varphi=\Delta \mu+K_{2}(\boldsymbol{u}, \varphi) \quad \text { in }\left(H^{1}\right)^{\prime}, \text { a.e. in }(0, T) \\
& \mu=a \varphi-J * \varphi+F^{\prime}(\varphi) \quad \text { a.e. in } \Omega \times(0, T)
\end{aligned}
$$

Remark 2. The pressure is excluded from (3.8) as usual; in fact, one (formally) has

$$
\mu \nabla \varphi=\nabla\left(F(\varphi)+\frac{a}{2} \varphi^{2}-(J * \varphi) \varphi\right)-\frac{\nabla a}{2} \varphi^{2}+(\nabla J * \varphi) \varphi .
$$


This explains that $\mu \nabla \varphi=K_{1}(\varphi)$ in $V_{p}^{\prime}$. Moreover, we recall that the conservation of mass holds, namely

$$
(\varphi, 1)=\left(\varphi_{0}, 1\right), \quad \forall t \in[0, T]
$$

Let us state first the existence of a weak solution.

Theorem 1. Let $p \geq 11 / 5$ and $q \geq q_{p}$ where $q_{p}$ is given by

$$
q_{p}:=\frac{2(3-p)}{5 p-6}, \quad \text { if } p<3
$$

$q_{p}>0$ arbitrary if $p=3$, and $q_{p}=0$ if $p>3$. If $\boldsymbol{h} \in L^{p^{\prime}}\left(0, T ; V_{p}^{\prime}\right), \boldsymbol{u}_{0} \in G, \varphi_{0} \in L^{2}(\Omega)$ with $\int_{\Omega} F\left(\varphi_{0}\right) d x<\infty$, then there exists a weak solution in the sense of Definition 1 satisfying the energy identity

$$
\mathcal{E}_{t o t}(\boldsymbol{u}(t), \varphi(t))+\int_{0}^{t}(\mathcal{S}(D \boldsymbol{u}), D \boldsymbol{u}) d \tau+\int_{0}^{t}\|\nabla \mu\|^{2} d \tau=\mathcal{E}_{t o t}\left(\boldsymbol{u}_{0}, \varphi_{0}\right)+\int_{0}^{t}\langle\boldsymbol{h}, \boldsymbol{u}\rangle_{V_{p}} d \tau,
$$

for all $t \in[0, T]$, where $\mathcal{E}_{\text {tot }}(\boldsymbol{u}, \varphi):=\frac{1}{2}\|\boldsymbol{u}\|^{2}+\mathcal{E}(\varphi)$, and $\mathcal{E}$ is given by (1.7).

The time regularity of a weak solution is given by

Theorem 2. Let $p>11 / 5, q \geq q_{p}$ and $\boldsymbol{h} \in N^{\kappa, p^{\prime}}\left(0, T ; V_{p}^{\prime}\right), \kappa>\delta_{\text {uniq }}$, where

$$
\delta_{\text {uniq }}=\max \left\{0,(p-1)\left(\frac{5}{2 p}-1\right)\right\} .
$$

Let $[\boldsymbol{u}, \varphi]$ be an arbitrary weak solution. Then $\boldsymbol{u} \in L^{p_{\text {uniq }}}\left(t_{0}, T ; V_{p}\right)$, whenever $t_{0} \in[0, T)$ be such that $\boldsymbol{u}\left(t_{0}\right) \in V_{p}$ and $t_{0}$ be a Lebesgue point of the function $t \mapsto\|\boldsymbol{h}(t)\|_{V_{p}^{\prime}}^{p^{\prime}}$. In particular, for any weak solution, one has $\boldsymbol{u} \in L_{\text {loc }}^{p_{\text {uniq }}}\left(0, T ; V_{p}\right)$.

Two consequences of Theorem 2 are the following

Corollary 1. Let the assumptions of Theorem 2 hold with $q>1 / 2$. Consider two weak solutions $\left[\boldsymbol{u}_{1}, \varphi_{1}\right],\left[\boldsymbol{u}_{2}, \varphi_{2}\right]$ and suppose that they coincide on some interval $\left[t_{0}, t_{0}+\tau\right] \subset$ $[0, T), \tau \in\left(0, T-t_{0}\right)$. Then the solutions coincide on $\left[t_{0}, T\right]$.

Corollary 2. Let the assumptions of Theorem 2 hold with $q>1 / 2$. Consider an arbitrary weak solution $[\boldsymbol{u}, \varphi]$. Suppose there exists $t_{0} \in[0, T)$ such that $\boldsymbol{u}\left(t_{0}\right) \in V_{p}, \varphi\left(t_{0}\right) \in L^{\infty}(\Omega)$ and $\boldsymbol{h} \in N^{\delta / p^{\prime}, p^{\prime}}\left(t_{0}, T ; V_{p}^{\prime}\right)$, where $\delta / p^{\prime} \geq \delta_{\text {uniq. }}$. Suppose, in addition, that $t_{0}$ is a Lebesgue point of the function $t \mapsto\|\boldsymbol{h}(t)\|_{V_{p}^{\prime}}^{p^{\prime}}$. Then

$$
\begin{aligned}
& \boldsymbol{u} \in N^{\frac{\delta}{2}, \infty}\left(t_{0}, T ; G\right) \cap N^{\frac{\delta}{p}, p}\left(t_{0}, T ; V_{p}\right) \cap N^{\frac{\delta}{2}, 2}\left(t_{0}, T ; V_{2}\right) \\
& \varphi \in N^{\frac{\delta}{2 q+q}, 2 q+2}\left(t_{0}, T ; L^{2 q+2}(\Omega)\right) \cap N^{\frac{\delta}{2}, 2}\left(t_{0}, T ; L^{2}(\Omega)\right) \cap L^{\infty}(\Omega \times(0, T)) .
\end{aligned}
$$

Theorem 2 and the related corollaries will be proven in Section 6, taking advantage of some auxiliary estimates derived in Section 5. In the next section a detailed proof of Theorem 1 is given. 


\section{Proof of Theorem 1}

The proof will be carried out by means of a Faedo-Galerkin approximation procedure, assuming at first that $\varphi_{0} \in D(B)=\left\{v \in H^{2}(\Omega): \partial_{\boldsymbol{n}} v=0\right.$ on $\left.\partial \Omega\right\}$, where $B=-\Delta+I$. The general case $\varphi_{0} \in L^{2}(\Omega)$ with $F\left(\varphi_{0}\right) \in L^{1}(\Omega)$ can be dealt in the same fashion as in [11], by means of a density argument and by relying on the form of the potential $F$ as a quadratic perturbation of a convex function.

In order to implement the approximation scheme we introduce the auxiliary Hilbert space $W_{s}$ defined by (cf. [29])

$$
W_{s}:=\overline{\mathcal{V}}^{H^{s}(\Omega)^{3}},
$$

where $s \geq 0$ is fixed. In particular, we shall assume $s \geq s_{p}$, where $s_{p}=5 / 2-3 / p$. This choice ensures that the following embeddings hold

$$
W_{s} \hookrightarrow V_{p} \hookrightarrow G \hookrightarrow V_{p}^{\prime} \hookrightarrow W_{s}^{\prime} .
$$

As a Galerkin base in $V_{p}$ we employ the family $\left\{\boldsymbol{w}_{j}\right\}_{j \geq 1}$, where each $\boldsymbol{w}_{j}$ solves

$$
\left(\boldsymbol{w}_{j}, \boldsymbol{v}\right)_{W_{s}}=\mu_{j}\left(\boldsymbol{w}_{j}, \boldsymbol{v}\right), \quad \forall \boldsymbol{v} \in W_{s} .
$$

In $V$ we choose as Galerkin base the family $\left\{\psi_{j}\right\}_{j \geq 1}$, where $\psi_{j}$ are the eigenfunctions of the operator $B$. Let $\mathcal{W}_{n}:=\left\langle\boldsymbol{w}_{1}, \cdots, \boldsymbol{w}_{n}\right\rangle$ and $\Psi_{n}:=\left\langle\psi_{1}, \cdots, \psi_{n}\right\rangle$ be the $n$-dimensional subspaces spanned by the first $n$ vectors of each base and let $\widetilde{P}_{n}$ and $P_{n}$ be the corresponding orthogonal projectors in $G$ and in $L^{2}(\Omega)$, respectively.

We look for three functions of the form

$$
\boldsymbol{u}_{n}(t)=\sum_{k=1}^{n} a_{k}^{(n)}(t) \boldsymbol{w}_{k}, \quad \varphi_{n}(t)=\sum_{k=1}^{n} b_{k}^{(n)}(t) \psi_{k}, \quad \mu_{n}(t)=\sum_{k=1}^{n} c_{k}^{(n)}(t) \psi_{k}
$$

which solve the following approximate problem

$$
\begin{aligned}
& \left(\boldsymbol{u}_{n}^{\prime}, \boldsymbol{w}\right)+\left(\mathcal{S}\left(D \boldsymbol{u}_{n}\right), D \boldsymbol{w}\right)+b\left(\boldsymbol{u}_{n}, \boldsymbol{u}_{n}, \boldsymbol{w}\right)=-\left(\varphi_{n} \nabla \mu_{n}, \boldsymbol{w}\right)+\left(\boldsymbol{h}_{n}, \boldsymbol{w}\right) \\
& \left(\varphi_{n}^{\prime}, \psi\right)+\left(\nabla \mu_{n}, \nabla \psi\right)=\left(\boldsymbol{u}_{n} \varphi_{n}, \nabla \psi\right) \\
& \mu_{n}=P_{n}\left(a \varphi_{n}-J * \varphi_{n}+F^{\prime}\left(\varphi_{n}\right)\right) \\
& \boldsymbol{u}_{n}(0)=\boldsymbol{u}_{0 n}, \quad \varphi_{n}(0)=\varphi_{0 n},
\end{aligned}
$$

for every $\boldsymbol{w} \in \mathcal{W}_{n}$ and every $\psi \in \Psi_{n}$, where $\boldsymbol{u}_{0 n} \in \mathcal{W}_{n}$ are such that $\boldsymbol{u}_{0 n} \rightarrow \boldsymbol{u}_{0}$ in $G$ and $\varphi_{0 n}=P_{n} \varphi_{0}$ (primes denote classical derivatives with respect to time). In (4.1) $\boldsymbol{h}_{n}$ denotes a sequence in $C^{0}([0, T] ; G)$ such that $\boldsymbol{h}_{n} \rightarrow \boldsymbol{h}$ in $L^{p^{\prime}}\left(0, T ; V_{p}^{\prime}\right)$. Here, we have denoted by $b(\cdot, \cdot, \cdot)$ the usual trilinear form which is employed in the theory of the incompressible Navier-Stokes system (cf. [35]), namely

$$
b(\boldsymbol{u}, \boldsymbol{z}, \boldsymbol{v}):=\int_{\Omega}(\boldsymbol{u} \cdot \nabla) \boldsymbol{z} \cdot \boldsymbol{v} \quad \forall \boldsymbol{u}, \boldsymbol{z}, \boldsymbol{v} \in V_{p} .
$$

In particular, we have $b(\boldsymbol{u}, \boldsymbol{u}, \boldsymbol{v})=-\left\langle K_{0}(\boldsymbol{u}), \boldsymbol{v}\right\rangle$. It is easy to see that this approximate problem can be solved by solving a Cauchy problem for a system of ordinary differential equations in the $2 n$ unknowns $a_{i}^{(n)}, b_{i}^{(n)}, i=1, \cdots, n$. Recalling that $F \in C^{1}(\mathbb{R})$ and $\mathcal{S} \in C^{0}\left(\mathbb{R}^{3 \times 3}\right)^{3 \times 3}$, Peano's theorem ensures that there exists $T_{n}^{*} \in(0,+\infty]$ such that this system admits a maximal solution $\mathbf{a}^{(n)}:=\left(a_{1}^{(n)}, \cdots, a_{n}^{(n)}\right), \mathbf{b}^{(n)}:=\left(b_{1}^{(n)}, \cdots, b_{n}^{(n)}\right)$ on $\left[0, T_{n}^{*}\right)$ satisfying $\mathbf{a}^{(n)}, \mathbf{b}^{(n)} \in C^{1}\left(\left[0, T_{n}^{*}\right) ; \mathbb{R}^{n}\right)$. Notice that, at this stage, the smoothness of $F$ and $\mathcal{S}$ are enough to guarantee the existence of a maximal solution $\mathbf{a}^{(n)}, \mathbf{b}^{(n)}$. 


\section{A priori estimates}

We now derive some basic energy estimates for the sequence of the approximate solutions $\boldsymbol{u}_{n}, \varphi_{n}$ and for the sequence of $\mu_{n}$.

We take $\boldsymbol{u}_{n}$ as test function in (4.1), $\mu_{n}$ as test function in (4.2) and we recall that $b\left(\boldsymbol{u}_{n}, \boldsymbol{u}_{n}, \boldsymbol{u}_{n}\right)=0$. By summing the ensuing identities we get

$$
\frac{d}{d t}\left(\frac{1}{2}\left\|\boldsymbol{u}_{n}\right\|^{2}+\mathcal{E}\left(\varphi_{n}\right)\right)+\left(\mathcal{S}\left(D \boldsymbol{u}_{n}\right), D \boldsymbol{u}_{n}\right)+\left\|\nabla \mu_{n}\right\|^{2}=\left(\boldsymbol{h}_{n}, \boldsymbol{u}_{n}\right),
$$

where the functional $\mathcal{E}$ is given by (1.7). By arguing as in [11] once more and using the coercivity assumption on the nonlinear function $\mathcal{S}$ (cf. (2.1)), the conditions on $F$, and the fact that, since $\varphi_{0} \in D(B)$, we have $\varphi_{0 n} \rightarrow \varphi_{0}$ in $H^{2}(\Omega)$ and hence also in $L^{\infty}(\Omega)$, we first deduce that $T_{n}^{*}=+\infty$ for every $n$, i.e., each approximate problem has a global in time solution, and furthermore we obtain the following estimates which hold for every $T \in(0,+\infty)$

$$
\begin{aligned}
& \left\|\boldsymbol{u}_{n}\right\|_{L^{\infty}(0, T ; G) \cap L^{p}\left(0, T ; V_{p}\right)} \leq N \\
& \left\|\varphi_{n}\right\|_{L^{\infty}\left(0, T ; L^{2 q+2}(\Omega)\right) \cap L^{2}\left(0, T ; H^{1}(\Omega)\right)} \leq N \\
& \left\|\mu_{n}\right\|_{L^{2}\left(0, T ; H^{1}(\Omega)\right)} \leq N \\
& \left\|F\left(\varphi_{n}\right)\right\|_{L^{\infty}\left(0, T ; L^{1}(\Omega)\right)} \leq N,
\end{aligned}
$$

where we have set $N=c M^{1 / 2}+c\|\boldsymbol{h}\|_{L^{p^{\prime}\left(0, T ; V_{p}^{\prime}\right)}}$, with $M$ given by

$$
M=c\left(1+\left\|\boldsymbol{u}_{0}\right\|^{2}+\left\|\varphi_{0}\right\|^{2}+\int_{\Omega} F\left(\varphi_{0}\right)\right) .
$$

The growth condition on the nonlinear function $\mathcal{S}$ (see (2.1)) as well as estimate (4.6) also provide a bound for the sequence of the nonlinear terms $\mathcal{S}\left(D \boldsymbol{u}_{n}\right)$. Indeed, we have

$$
\left\|\mathcal{S}\left(D \boldsymbol{u}_{n}\right)\right\|_{L^{p^{\prime}\left(0, T ; L^{p^{\prime}}(\Omega)^{3 \times 3}\right)} \leq C,}
$$

where, here and henceforth, $C$ will denote a positive constant depending on the norms of $\boldsymbol{u}_{0}, \varphi_{0}$ and $\boldsymbol{h}$ (cf. the constant $N$ in (4.6)-(4.8)). As far as the bound for the nonlinear terms $F^{\prime}\left(\varphi_{n}\right)$ in (4.3) is concerned, on account of (2.2) and (4.9), we immediately get

$$
\left\|F^{\prime}\left(\varphi_{n}\right)\right\|_{L^{\infty}\left(0, T ; L^{r}(\Omega)\right)} \leq C \text {. }
$$

The last estimates we need are for the sequences of the time derivatives $\boldsymbol{u}_{n}^{\prime}, \varphi_{n}^{\prime}$. Let us begin with the former one. Let us take $\boldsymbol{w} \in W_{s}$, and decompose it as $\boldsymbol{w}=\boldsymbol{w}_{I}+\boldsymbol{w}_{I I}$, where $\boldsymbol{w}_{I} \in \mathcal{W}_{n}$ and $\boldsymbol{w}_{I I} \in \mathcal{W}_{n}^{\perp}$, and notice that $\boldsymbol{w}_{I}$ and $\boldsymbol{w}_{I I}$ are orthogonal also in $W_{s}$. Then, from (4.1) we can write

$$
\begin{aligned}
\left\langle\boldsymbol{u}_{n}^{\prime}, \boldsymbol{w}\right\rangle_{W_{s}}=\left\langle\boldsymbol{u}_{n}^{\prime}, \boldsymbol{w}_{I}\right\rangle_{W_{s}} & =-\left(\mathcal{S}\left(D \boldsymbol{u}_{n}\right), D \boldsymbol{w}_{I}\right)-b\left(\boldsymbol{u}_{n}, \boldsymbol{u}_{n}, \boldsymbol{w}_{I}\right) \\
& -\left(\varphi_{n} \nabla \mu_{n}, \boldsymbol{w}_{I}\right)+\left(\boldsymbol{h}_{n}, \boldsymbol{w}_{I}\right) .
\end{aligned}
$$

Let us now estimate each of the four terms on the right hand side. As far as the first term is concerned, we have

$$
\begin{aligned}
& \left|\left(\mathcal{S}\left(D \boldsymbol{u}_{n}\right), D \boldsymbol{w}_{I}\right)\right| \leq \|\left(\mathcal{S}\left(D \boldsymbol{u}_{n}\right)\left\|_{\left(L^{p^{\prime}}\right)^{3 \times 3}}\right\| \boldsymbol{w}_{I} \|_{V_{p}}\right. \\
& \leq c\left(1+\left\|D \boldsymbol{u}_{n}\right\|_{\left(L^{p}\right)^{3 \times 3}}^{p-1}\right)\left\|\boldsymbol{w}_{I}\right\|_{W_{s}} \leq c\left(1+\left\|D \boldsymbol{u}_{n}\right\|_{\left(L^{p}\right)^{3 \times 3}}^{p-1}\right)\|\boldsymbol{w}\|_{W_{s}} .
\end{aligned}
$$

Concerning the convective term on the right hand side of (4.12), the simplest way to estimate it, which is enough for our purposes, is the following. Take $s>5 / 2$. This condition, 
which will be assumed henceforth, ensures that $\nabla \boldsymbol{w} \in H^{s-1}(\Omega)^{3 \times 3} \hookrightarrow L^{\infty}(\Omega)^{3 \times 3}$ (notice that the Hilbert space $W_{s}$ has just the role of an auxiliary space; hence $s$ can be suitably chosen). Thus we find

$$
\begin{aligned}
\left|b\left(\boldsymbol{u}_{n}, \boldsymbol{u}_{n}, \boldsymbol{w}_{I}\right)\right| & =\left|b\left(\boldsymbol{u}_{n}, \boldsymbol{w}_{I}, \boldsymbol{u}_{n}\right)\right| \leq\left\|\boldsymbol{u}_{n}\right\|^{2}\left\|\nabla \boldsymbol{w}_{I}\right\|_{\left(L^{\infty}\right)^{3 \times 3}} \\
& \leq c\left\|\boldsymbol{u}_{n}\right\|^{2}\left\|\boldsymbol{w}_{I}\right\|_{W_{s}} \leq c\left\|\boldsymbol{u}_{n}\right\|^{2}\|\boldsymbol{w}\|_{W_{s}} .
\end{aligned}
$$

Next, the contributions arising from the Korteweg and from the external force terms on the right hand side of (4.12) can be estimated as follows

$$
\begin{aligned}
& \left|\left(\varphi_{n} \nabla \mu_{n}, \boldsymbol{w}_{I}\right)\right| \leq\left\|\varphi_{n}\right\|\left\|\nabla \mu_{n}\right\|\left\|\boldsymbol{w}_{I}\right\|_{\left(L^{\infty}\right)^{3}} \leq c\left\|\varphi_{n}\right\|\left\|\nabla \mu_{n}\right\|\|\boldsymbol{w}\|_{W_{s}} \\
& \left|\left\langle\boldsymbol{h}_{n}, \boldsymbol{w}_{I}\right\rangle_{W_{s}}\right| \leq\left\|\boldsymbol{h}_{n}\right\|_{V_{p}^{\prime}}\left\|\boldsymbol{w}_{I}\right\|_{V_{p}} \leq c\left\|\boldsymbol{h}_{n}\right\|_{V_{p}^{\prime}}\|\boldsymbol{w}\|_{W_{s}} .
\end{aligned}
$$

Inserting estimates (4.13)-(4.16) into (4.12) we get

$$
\left\|\boldsymbol{u}_{n}^{\prime}\right\|_{W_{s}^{\prime}} \leq c\left(1+\left\|D \boldsymbol{u}_{n}\right\|_{\left(L^{p}\right)^{3 \times 3}}^{p-1}+\left\|\boldsymbol{u}_{n}\right\|^{2}+\left\|\varphi_{n}\right\|\left\|\nabla \mu_{n}\right\|+\left\|\boldsymbol{h}_{n}\right\|_{V_{p}^{\prime}}\right)
$$

and therefore, on account of the basic estimates (4.6)-(4.8) and of the fact that $\boldsymbol{h}_{n} \rightarrow \boldsymbol{h}$ in $L^{p^{\prime}}\left(0, T ; V_{p}^{\prime}\right)$, we immediately obtain the following bound for the sequence $\boldsymbol{u}_{n}^{\prime}$

$$
\left\|\boldsymbol{u}_{n}^{\prime}\right\|_{L^{p^{\prime}\left(0, T ; W_{s}^{\prime}\right)}} \leq C .
$$

In order to derive an estimate for the sequence of $\varphi_{n}^{\prime}$, take $\psi \in V$ and decompose it as $\psi=\psi_{I}+\psi_{I I}$, where $\psi_{I} \in \Psi_{n}$ and $\psi_{I I} \in \Psi_{n}^{\perp}$. Recall that $\psi_{I}$ and $\psi_{I I}$ are orthogonal also in $H^{1}(\Omega)$. Then, from $(4.2)$ we deduce

$$
\left\langle\varphi_{n}^{\prime}, \psi\right\rangle_{H^{1}}=\left\langle\varphi_{n}^{\prime}, \psi_{I}\right\rangle_{H^{1}}=-\left(\nabla \mu_{n}, \nabla \psi_{I}\right)+\left(\boldsymbol{u}_{n} \varphi_{n}, \nabla \psi_{I}\right)
$$

and the only term here which requires some care is the last one on the right hand side. This can be estimated as

$$
\begin{aligned}
\left|\left(\boldsymbol{u}_{n} \varphi_{n}, \nabla \psi_{I}\right)\right| & \leq\left\|\boldsymbol{u}_{n}\right\|_{\left(L^{2(1+1 / q)}\right)^{3}}\left\|\varphi_{n}\right\|_{L^{2 q+2}}\left\|\psi_{I}\right\|_{H^{1}} \\
& \leq c\left\|\boldsymbol{u}_{n}\right\|_{V_{p}}\left\|\varphi_{n}\right\|_{L^{2 q+2}}\|\psi\|_{H^{1}},
\end{aligned}
$$

where we have used condition $q \geq q_{p}$ (cf. (3.13)), which ensures that $2(1+1 / q) \leq$ $3 p /(3-p)$, and the Sobolev embedding $W^{1, p}(\Omega) \hookrightarrow L^{3 p /(3-p)}(\Omega)$ (let us assume, here and henceforth, to be in the worst case $p<3)$. Hence, we deduce

$$
\left\|\varphi_{n}^{\prime}\right\|_{\left(H^{1}\right)^{\prime}} \leq c\left(\left\|\nabla \mu_{n}\right\|+\left\|\boldsymbol{u}_{n}\right\|_{V_{p}}\left\|\varphi_{n}\right\|_{L^{2 q+2}}\right),
$$

which, on account of (4.6)-(4.8) again, entails the following estimate for the sequence of the time derivatives $\varphi_{n}^{\prime}$

$$
\left\|\varphi_{n}^{\prime}\right\|_{L^{2}\left(0, T ; H^{1}(\Omega)^{\prime}\right)} \leq C .
$$

Passage to the limit by compactness

On account of estimates (4.6)-(4.8), (4.10), (4.11), (4.17), (4.19), and owing to the AubinLions Lemma, we deduce that there exist

$$
\begin{aligned}
& \boldsymbol{u} \in L^{\infty}(0, T ; G) \cap L^{p}\left(0, T ; V_{p}\right) \\
& \varphi \in L^{\infty}\left(0, T ; L^{2 q+2}(\Omega)\right) \cap L^{2}\left(0, T ; H^{1}(\Omega)\right) \\
& \mu \in L^{2}\left(0, T ; H^{1}(\Omega)\right) \\
& \boldsymbol{\chi} \in L^{p^{\prime}}\left(0, T ; L^{p^{\prime}}(\Omega)^{3 \times 3}\right)
\end{aligned}
$$


with

$$
\begin{aligned}
& \frac{d}{d t} \boldsymbol{u} \in L^{p^{\prime}}\left(0, T ; W_{s}^{\prime}\right) \\
& \frac{d}{d t} \varphi \in L^{2}\left(0, T ; H^{1}(\Omega)^{\prime}\right),
\end{aligned}
$$

such that, for a not relabeled subsequence, we have

$$
\begin{aligned}
& \boldsymbol{u}_{n} \rightarrow \boldsymbol{u} \quad \text { weakly* in } L^{\infty}(0, T ; G) \\
& \boldsymbol{u}_{n} \rightarrow \boldsymbol{u} \quad \text { weakly in } L^{p}\left(0, T ; V_{p}\right) \\
& \boldsymbol{u}_{n} \rightarrow \boldsymbol{u} \quad \text { strongly in } L^{p}(0, T ; G) \text { and a.e. in } \Omega \times(0, T) \\
& \mathcal{S}\left(D \boldsymbol{u}_{n}\right) \rightarrow \chi \quad \text { weakly in } L^{p^{\prime}}\left(0, T ; L^{p^{\prime}}(\Omega)^{3 \times 3}\right) \\
& \boldsymbol{u}_{n}^{\prime} \rightarrow \frac{d}{d t} \boldsymbol{u} \quad \text { weakly in } L^{p^{\prime}}\left(0, T ; W_{s}^{\prime}\right) \\
& \varphi_{n} \rightarrow \varphi \quad \text { weakly* in } L^{\infty}\left(0, T ; L^{2 q+2}(\Omega)\right) \\
& \varphi_{n} \rightarrow \varphi \quad \text { weakly in } L^{2}\left(0, T ; H^{1}(\Omega)\right) \\
& \varphi_{n} \rightarrow \varphi \quad \text { strongly in } L^{2}\left(0, T ; L^{2}(\Omega)\right) \text { and a.e. in } \Omega \times(0, T) \\
& \varphi_{n}^{\prime} \rightarrow \frac{d}{d t} \varphi \quad \text { weakly in } L^{2}\left(0, T ; H^{1}(\Omega)^{\prime}\right) \\
& \mu_{n} \rightarrow \mu \quad \text { weakly in } L^{2}\left(0, T ; H^{1}(\Omega)\right) \\
& F^{\prime}\left(\varphi_{n}\right) \rightarrow F^{\prime}(\varphi) \quad \text { weakly in } L^{\infty}\left(0, T ; L^{r}(\Omega)\right) .
\end{aligned}
$$

By means of a standard argument, (4.26)-(4.35) allow to pass to the limit in (4.1)-(4.2), to deduce that the following weak formulation holds

$$
\begin{aligned}
& \left\langle\frac{d}{d t} \boldsymbol{u}, \boldsymbol{w}\right\rangle_{W_{s}}+(\chi, D \boldsymbol{w})+b(\boldsymbol{u}, \boldsymbol{u}, \boldsymbol{w})=-(\varphi \nabla \mu, \boldsymbol{w})+\langle\boldsymbol{h}, \boldsymbol{w}\rangle_{W_{s}} \\
& \left\langle\frac{d}{d t} \varphi, \psi\right\rangle_{H^{1}}+(\nabla \mu, \nabla \psi)=(\boldsymbol{u} \varphi, \nabla \psi),
\end{aligned}
$$

for every $\boldsymbol{w} \in W_{s}$ and every $\psi \in H^{1}(\Omega)$. In particular, we have used the convergence $b\left(\boldsymbol{u}_{n}, \boldsymbol{u}_{n}, \boldsymbol{w}\right) \rightarrow b(\boldsymbol{u}, \boldsymbol{u}, \boldsymbol{w})$ in $\mathcal{D}^{\prime}(0, T)$ as $n \rightarrow \infty$ for every $\boldsymbol{w} \in \mathcal{W}_{m}$, which can be easily proved from (4.28) (see [29]). Moreover, we claim that $\mu=a \varphi-J * \varphi+F^{\prime}(\varphi)$. Indeed, from (4.3), for every $v \in \Psi_{n}$ and every $k \geq n$ ( $n$ is fixed), we have

$$
\int_{0}^{T}\left(\mu_{k}(t), v\right) \chi(t) d t=\int_{0}^{T}\left(a \varphi_{k}-J * \varphi_{k}+F^{\prime}\left(\varphi_{k}\right), v\right) \chi(t) d t, \quad \forall \chi \in \mathcal{D}(0, T) .
$$

By passing to the limit as $k \rightarrow \infty$ in this identity and using the convergences (4.35), (4.33) (which implies $J * \varphi_{k} \rightarrow J * \varphi$ strongly in $L^{2}\left(0, T ; H^{1}(\Omega)\right)$ ) and (4.36), on account of the density of $\left\{\Psi_{n}\right\}_{n \geq 1}$ in $L^{2}(\Omega)$ we get the desired claim.

By means of a comparison argument in (4.37), we now show that the regularity for the time derivative $\frac{d}{d t} \boldsymbol{u}$ is actually better than (4.24). Indeed, notice first that, by using Sobolev embedding, the Korteweg force term can be estimated, for every $\boldsymbol{w} \in V_{p}$, as

$$
\begin{aligned}
|(\varphi \nabla \mu, \boldsymbol{w})| & \leq\|\varphi\|_{L^{6 p /(5 p-6)}}\|\nabla \mu\|\|\boldsymbol{w}\|_{\left(L^{3 p /(3-p)}\right)^{3}} \\
& \leq C\|\nabla \mu\|\|\boldsymbol{w}\|_{V_{p}},
\end{aligned}
$$


where we have used the fact that, since $q \geq q_{p}$, then $\varphi \in L^{\infty}\left(0, T ; L^{6 p /(5 p-6)}(\Omega)\right)$, thanks to the regularity (4.21). Moreover, the kinetic term in (4.37) is estimated as usual, by means of Gagliardo-Nirenberg inequality, to give

$$
\begin{aligned}
|b(\boldsymbol{u}, \boldsymbol{u}, \boldsymbol{w})| & \leq c\|\boldsymbol{u}\|_{\left(L^{3 p /(4 p-6))^{3}}\right.}\|\boldsymbol{u}\|_{V_{p}}\|\boldsymbol{w}\|_{\left(L^{3 p /(3-p))^{3}}\right.} \\
& \leq c\|\boldsymbol{u}\|_{V_{p}}^{6 /(5 p-6)}\|\boldsymbol{w}\|_{V_{p}} .
\end{aligned}
$$

By comparison in (4.37) we therefore get

$$
\left|\left\langle\frac{d}{d t} \boldsymbol{u}, \boldsymbol{w}\right\rangle_{V_{p}}\right| \leq c\left(\|\boldsymbol{\chi}\|_{\left(L^{p^{\prime}}\right)^{3 \times 3}}+\|\boldsymbol{u}\|_{V_{p}}^{6 /(5 p-6)}+\|\nabla \mu\|+\|\boldsymbol{h}\|_{V_{p}^{\prime}}\right)\|\boldsymbol{w}\|_{V_{p}},
$$

for all $\boldsymbol{w} \in W_{s}$. This allows to conclude that $\frac{d}{d t} \boldsymbol{u}(t)$ can be continuously extended to $V_{p}$, for almost every $t>0$, and, since we have $p \geq 11 / 5$, that there holds

$$
\frac{d}{d t} \boldsymbol{u} \in L^{p^{\prime}}\left(0, T ; V_{p}^{\prime}\right)
$$

Moreover, the weak formulation (4.37) holds also for every $\boldsymbol{w} \in V_{p}$.

To complete the proof, there remains to prove that $(\boldsymbol{\chi}, D \boldsymbol{w})=(\mathcal{S}(D \boldsymbol{u}), D \boldsymbol{w})$, for all $\boldsymbol{w} \in V_{p}$, or, equivalently that $\operatorname{div} \boldsymbol{\chi}=\operatorname{div} \mathcal{S}(D \boldsymbol{u})$ in $\mathcal{D}^{\prime}(\Omega)^{3}$. This identification will be achieved by means of a monotonicity argument.

\section{Identification $\operatorname{div} \boldsymbol{\chi}=\operatorname{div} \mathcal{S}(D \boldsymbol{u})$ by monotonicity}

We first see that the following energy identity holds

$$
\begin{aligned}
& \frac{1}{2}\|\boldsymbol{u}(t)\|^{2}+\mathcal{E}(\varphi(t))+\int_{0}^{t}(\boldsymbol{\chi}, D \boldsymbol{u}) d \tau+\int_{0}^{t}\|\nabla \mu\|^{2} d \tau \\
& =\frac{1}{2}\left\|\boldsymbol{u}_{0}\right\|^{2}+\mathcal{E}\left(\varphi_{0}\right)+\int_{0}^{t}\langle\boldsymbol{h}, \boldsymbol{u}\rangle_{V_{p}} d \tau, \quad \forall t \in[0, T] .
\end{aligned}
$$

Indeed, we can take $\boldsymbol{w}=\boldsymbol{u}(\tau)$ as test function in (4.37) and $\psi=\mu(\tau)$ as test function in (4.38). Notice that this is allowed, on account of (4.41) and of (4.25), respectively. We then employ [12, Proposition 4.2.] in order to rewrite the term $\left\langle\frac{d}{d t} \varphi, F^{\prime}(\varphi)\right\rangle_{H^{1}}$, as well as the standard identity $\left\langle\frac{d}{d t} \boldsymbol{u}, \boldsymbol{u}\right\rangle_{V_{p}}=\frac{1}{2} \frac{d}{d t}\|\boldsymbol{u}\|^{2}$ (see, e.g., [35, Lemma 1.3, Chapter III]).

By adding and integrating the resulting identities between 0 and $t$, we get (4.42).

Take now an arbitrary $\boldsymbol{v} \in L^{p}\left(0, T ; V_{p}\right)$ and set, for all $t \in[0, T]$,

$$
\begin{aligned}
Z_{n}(t) & :=\frac{1}{2}\left\|\boldsymbol{u}_{n}(t)\right\|^{2}+\int_{0}^{t}\left(\mathcal{S}\left(D \boldsymbol{u}_{n}\right)-\mathcal{S}(D \boldsymbol{v}), D \boldsymbol{u}_{n}-D \boldsymbol{v}\right) d \tau \\
& +\int_{0}^{t}\left\|\nabla\left(\mu_{n}-\mu\right)\right\|^{2} d \tau+\mathcal{E}\left(\varphi_{n}(t)\right)
\end{aligned}
$$

Using the energy identity for the approximate solutions obtained by integrating (4.5) between 0 and $t$, we immediately see that $Z_{n}(t)$ can be rewritten in the form

$$
\begin{aligned}
Z_{n}(t) & =\frac{1}{2}\left\|\boldsymbol{u}_{0 n}\right\|^{2}+\mathcal{E}\left(\varphi_{0 n}\right)+\int_{0}^{t}\left\langle\boldsymbol{h}_{n}, \boldsymbol{u}_{n}\right\rangle_{V_{p}} d \tau-\int_{0}^{t}\left(\mathcal{S}\left(D \boldsymbol{u}_{n}\right), D \boldsymbol{v}\right) d \tau \\
& -\int_{0}^{t}(\mathcal{S}(D \boldsymbol{v}), D \boldsymbol{u}-D \boldsymbol{v}) d \tau+\int_{0}^{t}\|\nabla \mu\|^{2} d \tau-2 \int_{0}^{t}\left(\nabla \mu_{n}, \nabla \mu\right) d \tau .
\end{aligned}
$$


By employing the convergences above, we get

$$
\begin{aligned}
Z_{n}(t) & \rightarrow \frac{1}{2}\left\|\boldsymbol{u}_{0}\right\|^{2}+\mathcal{E}\left(\varphi_{0}\right)+\int_{0}^{t}\langle\boldsymbol{h}, \boldsymbol{u}\rangle_{V_{p}} d \tau-\int_{0}^{t}(\boldsymbol{\chi}, D \boldsymbol{v}) d \tau \\
& -\int_{0}^{t}(\mathcal{S}(D \boldsymbol{v}), D \boldsymbol{u}-D \boldsymbol{v}) d \tau-\int_{0}^{t}\|\nabla \mu\|^{2} d \tau
\end{aligned}
$$

On the other hand, thanks to the monotonicity of the map $\mathcal{S}: \mathbb{R}^{3 \times 3} \rightarrow \mathbb{R}^{3 \times 3}$ (see (2.1)), to the lower semicontinuity of the norms and to Fatou's Lemma, we have

$$
\liminf _{n \rightarrow \infty} Z_{n}(t) \geq \frac{1}{2}\|\boldsymbol{u}(t)\|^{2}+\mathcal{E}(\varphi(t))
$$

Hence, we obtain

$$
\begin{aligned}
\frac{1}{2}\|\boldsymbol{u}(t)\|^{2}+\mathcal{E}(\varphi(t)) & \leq \frac{1}{2}\left\|\boldsymbol{u}_{0}\right\|^{2}+\mathcal{E}\left(\varphi_{0}\right)+\int_{0}^{t}\langle\boldsymbol{h}, \boldsymbol{u}\rangle_{V_{p}} d \tau-\int_{0}^{t}(\boldsymbol{\chi}, D \boldsymbol{v}) d \tau \\
& -\int_{0}^{t}(\mathcal{S}(D \boldsymbol{v}), D \boldsymbol{u}-D \boldsymbol{v}) d \tau-\int_{0}^{t}\|\nabla \mu\|^{2} d \tau
\end{aligned}
$$

which, combined with (4.42), yields the variational inequality

$$
\int_{0}^{t}(\chi-\mathcal{S}(D \boldsymbol{v}), D \boldsymbol{u}-D \boldsymbol{v}) d \tau \geq 0 \quad \forall \boldsymbol{v} \in L^{p}\left(0, T ; V_{p}\right) .
$$

The well-known Minty trick (take $\boldsymbol{v}=\boldsymbol{u}+\epsilon \boldsymbol{z}$, with $\boldsymbol{z} \in L^{p}\left(0, T ; V_{p}\right)$ arbitrary and pass to the limit as $\epsilon \rightarrow 0$ ), taking also into account the continuity of $\mathcal{S}$, combined with a density argument (take $\boldsymbol{z}=\eta \boldsymbol{w}$, where $\boldsymbol{w} \in V_{p}$ and $\eta \in \mathcal{D}(0, T)$ are arbitrary) allow us to deduce that $(\boldsymbol{\chi}, D \boldsymbol{w})=(\boldsymbol{S}(D \boldsymbol{u}), D \boldsymbol{w})$, for all $\boldsymbol{w} \in V_{p}$, i.e., the required identification.

\section{Preliminary estimates}

We will henceforth assume that the assumptions of Theorem 1 are satisfied so that we can consider a weak solution $[\boldsymbol{u}, \varphi]$.

Lemma 2. Let $p \geq 11 / 5, q \geq q_{p}$. Then any weak solution $[\boldsymbol{u}, \varphi]$ satisfies

$$
\begin{aligned}
\left\|\frac{d}{d t} \boldsymbol{u}(t)\right\|_{V_{p}^{\prime}} & \leq C\left(1+\|\boldsymbol{u}(t)\|_{V_{p}}^{p-1}+\|\boldsymbol{h}(t)\|_{V_{p}^{\prime}}\right) \\
\left\|\frac{d}{d t} \varphi(t)\right\|_{\left(H^{1}\right)^{\prime}} & \leq C\left(\|\mu(t)\|_{H^{1}}+\|\boldsymbol{u}(t)\|_{V_{p}}\right)
\end{aligned}
$$

where $C>0$ depends on the initial data (in particular, on $\left\|\boldsymbol{u}_{0}\right\|,\left\|\varphi_{0}\right\|$ and on $\int_{\Omega} F\left(\varphi_{0}\right)$ ), on $\|\boldsymbol{h}\|_{L^{p^{\prime}\left(0, T ; V_{p}^{\prime}\right)}}$ and on other known quantities. Here we take advantage of the energy identity (see (4.5)) to bound the $L^{\infty}$-norms of $\|\boldsymbol{u}(t)\|,\|\varphi(t)\|_{L^{2 q+2}}$.

Proof. Estimate (5.2) can be immediately obtained from the variational formulation (4.38) by arguing as in (4.18) in order to control the term on the right hand side. The regularity (4.21) is taken into account as well.

As far as (5.1) is concerned, instead of using (4.37), we can argue from the variational formulation (3.8), which is obtained by eliminating the chemical potential $\mu$ in the Korteweg force term. Notice that in the proof of Theorem 1 we employed a weak formulation 
of (1.13) with $\mu$ appearing explicitly in the Korteweg force. However, since $F$ is regular, it easy to see that this formulation is equivalent to (3.8).

Omitting the variable $t$ for simplicity, we take $\boldsymbol{v} \in V_{p}$ with $\|\boldsymbol{v}\|_{V_{p}} \leq 1$ and estimate

$$
\begin{aligned}
\left\langle\frac{d}{d t} \boldsymbol{u}, \boldsymbol{v}\right\rangle_{V_{p}} & =-\langle N(\boldsymbol{u}), \boldsymbol{v}\rangle_{V_{p}}+\left\langle K_{0}(\boldsymbol{u}), \boldsymbol{v}\right\rangle_{V_{p}}+\left\langle K_{1}(\varphi), \boldsymbol{v}\right\rangle_{V_{p}}+\langle\boldsymbol{h}, \boldsymbol{v}\rangle_{V_{p}} \\
& =D_{1}+D_{2}+D_{3}+\langle\boldsymbol{h}, \boldsymbol{v}\rangle_{V_{p}} .
\end{aligned}
$$

Clearly $\left|D_{1}\right| \leq C\left(1+\|\boldsymbol{u}\|_{V_{p}}^{p-1}\right)$ (cf. (3.1) and (4.13)). Using the interpolation

$$
\|v\|_{L^{2 p^{\prime}}} \leq\|v\|^{1-\gamma}\|v\|_{L^{p^{*}}}^{\gamma} \quad \gamma=\frac{3}{5 p-6}, \quad 1-\gamma=\frac{5 p-9}{5 p-6},
$$

we have (cf. (3.2))

$$
\left|D_{2}\right| \leq \int_{\Omega}|\boldsymbol{u}|^{2}|\nabla \boldsymbol{v}| d x \leq\|\boldsymbol{u}\|_{L^{2 p^{\prime}}}^{2}\|\nabla \boldsymbol{v}\|_{L^{p}} \leq\|\boldsymbol{u}\|^{2(1-\gamma)}\|\boldsymbol{u}\|_{V_{p}}^{2 \gamma}\|\boldsymbol{v}\|_{V_{p}} \leq C\left(1+\|\boldsymbol{u}\|_{V_{p}}^{p-1}\right) .
$$

Here we have used that $2 \gamma \leq p-1$, which is just $p \geq 11 / 5$. Finally, on account of (3.3), in order to estimate $D_{3}$ we just need to observe that

$$
\int_{\Omega}|\nabla J * \varphi\|\varphi\| \boldsymbol{v}| d x \leq\|\nabla J * \varphi\|_{L^{2\left(p^{*}\right)^{\prime}}}\|\varphi\|_{L^{2\left(p^{*}\right)^{\prime}}}\|\boldsymbol{v}\|_{L^{p^{*}}} \leq C,
$$

since we have $2\left(p^{*}\right)^{\prime}=6 p /(4 p-3) \leq 2 q+2$, as a consequence of the condition $q \geq q_{p}$.

The following lemma is concerned with the estimates of the time differences.

Lemma 3. Let $p \geq 11 / 5, q \geq q_{p}$ and let $[\boldsymbol{u}, \varphi]$ be an arbitrary weak solution.

(1) Let $t_{0} \in[0, T)$ be such that $\boldsymbol{u}\left(t_{0}\right) \in V_{p}$, and $t=t_{0}$ be a Lebesgue point of the function $t \mapsto\|\boldsymbol{h}(t)\|_{V_{p}^{\prime}}^{p^{\prime}}$. Then

$$
\left\|\boldsymbol{u}\left(t_{0}+h\right)-\boldsymbol{u}\left(t_{0}\right)\right\|^{2}+\left\|\varphi\left(t_{0}+h\right)-\varphi\left(t_{0}\right)\right\|_{\left(H^{1}\right)^{\prime}}^{2} \leq c h, \quad h \in\left(0, h_{0}\right)
$$

for some $c, h_{0}>0$ depending on $\boldsymbol{h}$ and $t_{0}$.

(2) Estimate (5.6) holds for almost every $t_{0} \in(0, T)$.

Proof. (1) We can write

$$
\begin{aligned}
& \left\|\boldsymbol{u}\left(t_{0}+h\right)-\boldsymbol{u}\left(t_{0}\right)\right\|^{2} \\
& =\left(\left\|\boldsymbol{u}\left(t_{0}+h\right)\right\|^{2}-\left\|\boldsymbol{u}\left(t_{0}\right)\right\|^{2}\right)+2\left(\boldsymbol{u}\left(t_{0}\right)-\boldsymbol{u}\left(t_{0}+h\right), \boldsymbol{u}\left(t_{0}\right)\right)=E_{1}+E_{2} .
\end{aligned}
$$

To estimate $E_{1}$, we test $(3.8)$ by $2 \boldsymbol{u}$ and integrate over $t \in(0, h)$. Observing that $\langle N(\boldsymbol{u}), \boldsymbol{u}\rangle_{V_{p}} \geq c_{2}\|\boldsymbol{u}\|_{V_{p}}^{p},\left\langle K_{0}(\boldsymbol{u}), \boldsymbol{u}\right\rangle_{V_{p}}=0$ and recalling (3.11), we get

$$
\begin{gathered}
\left|\left\langle K_{1}(\varphi), \boldsymbol{u}\right\rangle_{V_{p}}\right| \leq c\|\varphi\|_{L^{2\left(p^{*}\right)^{\prime}}}^{2}\|\boldsymbol{u}\|_{V_{p}} \leq c\|\varphi\|_{L^{2 q+2}}^{2}\|\boldsymbol{u}\|_{V_{p}} \leq \frac{c_{2}}{4}\|\boldsymbol{u}\|_{V_{p}}^{p}+c \\
\left|\langle\boldsymbol{h}, \boldsymbol{u}\rangle_{V_{p}}\right| \leq\|\boldsymbol{h}\|_{V_{p}^{\prime}}\|\boldsymbol{u}\|_{V_{p}} \leq \frac{c_{2}}{4}\|\boldsymbol{u}\|_{V_{p}}^{p}+c\|\boldsymbol{h}\|_{V_{p}^{\prime}}^{p^{\prime}}
\end{gathered}
$$

Here $p^{*}=3 p /(3-p)$ (recall that we are working in the worst case $p<3$ ). This yields

$$
E_{1} \leq-c_{2} \int_{t_{0}}^{t_{0}+h}\|\boldsymbol{u}(t)\|_{V_{p}}^{p} d t+c \int_{t_{0}}^{t_{0}+h}\|\boldsymbol{h}(t)\|_{V_{p}}^{p^{\prime}} d t+\mathcal{O}(h) .
$$


Here $c>0$ depends on the initial data, on $\|\boldsymbol{h}\|_{L^{p^{\prime}\left(0, T ; V_{p}^{\prime}\right)}}$ and on other known quantities through the energy estimate (see $(4.5))$. To estimate $E_{2}$, we write

$$
\begin{aligned}
\left|\left(\boldsymbol{u}\left(t_{0}\right)-\boldsymbol{u}\left(t_{0}+h\right), \boldsymbol{u}\left(t_{0}\right)\right)\right| & \leq \int_{t_{0}}^{t_{0}+h}\left|\left\langle\frac{d}{d t} \boldsymbol{u}(t), \boldsymbol{u}\left(t_{0}\right)\right\rangle_{V_{p}}\right| d t \\
& \leq \int_{t_{0}}^{t_{0}+h}\left\|\frac{d}{d t} \boldsymbol{u}(t)\right\|_{V_{p}^{\prime}}\left\|\boldsymbol{u}\left(t_{0}\right)\right\|_{V_{p}} d t \\
& \leq c \int_{t_{0}}^{t_{0}+h}\left(1+\|\boldsymbol{u}(t)\|_{V_{p}}^{p-1}+\|\boldsymbol{h}(t)\|_{V_{p}^{\prime}}\right) d t
\end{aligned}
$$

employing (5.1). This yields

$$
E_{2} \leq c_{2} \int_{t_{0}}^{t_{0}+h}\|\boldsymbol{u}(t)\|_{V_{p}}^{p} d t+c \int_{t_{0}}^{t_{0}+h}\left(1+\|\boldsymbol{h}(t)\|_{V_{p}}^{p^{\prime}}\right) d t
$$

with $c$ also depending on $\left\|\boldsymbol{u}\left(t_{0}\right)\right\|_{V_{p}}$. Combining (5.9) with (5.10) we get

$$
\left\|\boldsymbol{u}\left(t_{0}+h\right)-\boldsymbol{u}\left(t_{0}\right)\right\|^{2} \leq c \int_{t_{0}}^{t_{0}+h}\left(1+\|\boldsymbol{h}(t)\|_{V_{p}}^{p^{\prime}}\right) d t .
$$

Therefore $\boldsymbol{u}$ satisfies an estimate of the form (5.6) recalling that

$$
\limsup _{h \rightarrow 0+} \frac{1}{h} \int_{t_{0}-h}^{t_{0}+h}\|\boldsymbol{h}(t)\|_{V_{p}}^{p^{\prime}} d t<\infty
$$

at the Lebesgue point $t=t_{0}$. A similar estimate holds for $\left\|\varphi\left(t_{0}+h\right)-\varphi\left(t_{0}\right)\right\|_{\left(H^{1}\right)^{\prime}}^{2}$ for any $t_{0}$ since $\varphi \in W^{1,2}\left(0, T ; H^{1}(\Omega)^{\prime}\right) \hookrightarrow C^{0,1 / 2}\left(0, T ; H^{1}(\Omega)^{\prime}\right)$.

(2) It follows immediately as almost every $t_{0} \in(0, T)$ is a Lebesgue point (see the assumptions of (1)).

We can now evaluate the difference of two weak solutions as follows

Lemma 4. Let $p \geq 11 / 5, q \geq q_{p}$. Suppose that $\left[\boldsymbol{u}_{1}, \varphi_{1}\right],\left[\boldsymbol{u}_{2}, \varphi_{2}\right]$ are two weak solutions. Also, assume the additional regularity

$$
\begin{aligned}
& \boldsymbol{u}_{2} \in L^{p_{\text {uniq }}}\left(0, T ; V_{p}\right), \quad p_{\text {uniq }}=\frac{2 p}{2 p-3} \\
& \varphi_{1}, \varphi_{2} \in L^{P}\left(0, T ; L^{Q}(\Omega)\right), \quad P=\frac{2 Q}{Q-3}, \quad Q>3 .
\end{aligned}
$$

Then the following inequality holds

$$
\begin{aligned}
& \frac{d}{d t}\left(\left\|\boldsymbol{u}_{1}-\boldsymbol{u}_{2}\right\|^{2}+\left\|\varphi_{1}-\varphi_{2}\right\|_{\left(H^{1}\right)^{\prime}}^{2}+\left|\overline{\varphi_{1}-\varphi_{2}}\right|\right) \\
& +c\left(\left\|\boldsymbol{u}_{1}-\boldsymbol{u}_{2}\right\|_{V_{2}}^{2}+\left\|\boldsymbol{u}_{1}-\boldsymbol{u}_{2}\right\|_{V_{p}}^{p}+\left\|\varphi_{1}-\varphi_{2}\right\|^{2}+\left\|\varphi_{1}-\varphi_{2}\right\|_{L^{2 q+2}}^{2 q+2}\right) \\
& \leq m(t)\left(\left\|\boldsymbol{u}_{1}-\boldsymbol{u}_{2}\right\|^{2}+\left\|\varphi_{1}-\varphi_{2}\right\|_{\left(H^{1}\right)^{\prime}}^{2}+\left|\overline{\varphi_{1}-\varphi_{2}}\right|\right)
\end{aligned}
$$

where $m \in L^{1}(0, T)$ only depends on the norms of $\left[\boldsymbol{u}_{j}, \varphi_{j}\right], j=1,2$, related to (3.5)-(3.7) and on the norms of $\varphi_{1},\left[\boldsymbol{u}_{2}, \varphi_{2}\right]$ related to (5.12)-(5.13). 
Proof. Observe that

$$
\begin{aligned}
\frac{1}{2} \frac{d}{d t}\left\|\boldsymbol{u}_{1}-\boldsymbol{u}_{2}\right\|^{2} & =\left\langle\frac{d}{d t}\left(\boldsymbol{u}_{1}-\boldsymbol{u}_{2}\right), \boldsymbol{u}_{1}-\boldsymbol{u}_{2}\right\rangle_{V_{p}} \\
& =-\left\langle N\left(\boldsymbol{u}_{1}\right)-N\left(\boldsymbol{u}_{2}\right), \boldsymbol{u}_{1}-\boldsymbol{u}_{2}\right\rangle_{V_{p}}+\left\langle K_{0}\left(\boldsymbol{u}_{1}\right)-K_{0}\left(\boldsymbol{u}_{2}\right), \boldsymbol{u}_{1}-\boldsymbol{u}_{2}\right\rangle_{V_{p}} \\
& +\left\langle K_{1}\left(\varphi_{1}\right)-K_{1}\left(\varphi_{2}\right), \boldsymbol{u}_{1}-\boldsymbol{u}_{2}\right\rangle_{V_{p}}=D_{1}+D_{2}+D_{3} .
\end{aligned}
$$

Recalling (2.1), we have

$$
D_{1} \leq-c\left(\left\|\boldsymbol{u}_{1}-\boldsymbol{u}_{2}\right\|_{V_{2}}^{2}+\left\|\boldsymbol{u}_{1}-\boldsymbol{u}_{2}\right\|_{V_{p}}^{p}\right) .
$$

Then, using the interpolation

$$
\|v\|_{L^{\rho}} \leq\|v\|^{\theta}\|v\|_{L^{6}}^{1-\theta}, \quad \theta=\frac{2 p-3}{p}, \rho=\frac{6 p}{5 p-6},
$$

we find

$$
\begin{aligned}
\left|D_{2}\right| & \leq \int_{\Omega}\left|\boldsymbol{u}_{2}\right|\left|\boldsymbol{u}_{1}-\boldsymbol{u}_{2}\right|\left|\nabla\left(\boldsymbol{u}_{1}-\boldsymbol{u}_{2}\right)\right| d x \leq\left\|\boldsymbol{u}_{2}\right\|_{\left(L^{p^{*}}\right)^{3}}\left\|\boldsymbol{u}_{1}-\boldsymbol{u}_{2}\right\|_{\left(L^{\rho}\right)^{3}}\left\|\boldsymbol{u}_{1}-\boldsymbol{u}_{2}\right\|_{V_{2}} \\
& \leq\left\|\boldsymbol{u}_{2}\right\|_{V_{p}}\left\|\boldsymbol{u}_{1}-\boldsymbol{u}_{2}\right\|^{\theta}\left\|\boldsymbol{u}_{1}-\boldsymbol{u}_{2}\right\|_{V_{2}}^{2-\theta} \leq \delta\left\|\boldsymbol{u}_{1}-\boldsymbol{u}_{2}\right\|_{V_{2}}^{2}+C_{\delta}\left\|\boldsymbol{u}_{2}\right\|_{V_{p}}^{p_{\text {uniq }}}\left\|\boldsymbol{u}_{1}-\boldsymbol{u}_{2}\right\|^{2} .
\end{aligned}
$$

Concerning $D_{3}$, one writes

$$
\begin{aligned}
\left|D_{3}\right| & \leq \int_{\Omega}\left(\frac{|\nabla a|}{2}\left(\left|\varphi_{1}\right|+\left|\varphi_{2}\right|\right)\left|\varphi_{1}-\varphi_{2}\right|+\left|\varphi_{1}\right|\left|\nabla J *\left(\varphi_{1}-\varphi_{2}\right)\right|\right)\left|\boldsymbol{u}_{1}-\boldsymbol{u}_{2}\right| d x \\
& +\int_{\Omega}\left|\nabla J * \varphi_{2}\right|\left|\varphi_{1}-\varphi_{2}\right|\left|\boldsymbol{u}_{1}-\boldsymbol{u}_{2}\right| d x \\
& \leq \frac{3 b}{2}\left(\left\|\varphi_{1}\right\|_{L^{3+\varepsilon}}+\left\|\varphi_{2}\right\|_{L^{3+\varepsilon}}\right)\left\|\varphi_{1}-\varphi_{2}\right\|\left\|\boldsymbol{u}_{1}-\boldsymbol{u}_{2}\right\|_{\left(L^{6-\widetilde{\varepsilon}}\right)^{3}} \\
& \leq c\left(\left\|\varphi_{1}\right\|_{L^{3+\varepsilon}}+\left\|\varphi_{2}\right\|_{L^{3+\varepsilon}}\right)\left\|\varphi_{1}-\varphi_{2}\right\|\left\|\boldsymbol{u}_{1}-\boldsymbol{u}_{2}\right\|^{\gamma}\left\|\boldsymbol{u}_{1}-\boldsymbol{u}_{2}\right\|_{V_{2}}^{1-\gamma} \\
& \leq \delta\left(\left\|\varphi_{1}-\varphi_{2}\right\|^{2}+\left\|\boldsymbol{u}_{1}-\boldsymbol{u}_{2}\right\|_{V_{2}}^{2}\right)+C_{\delta}\left(\left\|\varphi_{1}\right\|_{L^{3+\varepsilon}}^{P}+\left\|\varphi_{2}\right\|_{L^{3+\varepsilon}}^{P}\right)\left\|\boldsymbol{u}_{1}-\boldsymbol{u}_{2}\right\|^{2} .
\end{aligned}
$$

In the second inequality we have used the fact that $\|\nabla J * \psi\|_{L^{p}} \leq b\|\psi\|_{L^{p}}$, for all $\psi \in L^{p}(\Omega)$ and for every $1 \leq p \leq \infty$. Morover, $\varepsilon>0$ is fixed arbitrary, $\widetilde{\varepsilon}>0$ is such that $(3+\varepsilon)^{-1}+(6-\widetilde{\varepsilon})^{-1}=1 / 2, \gamma:=\varepsilon /(3+\varepsilon), P:=2(3+\varepsilon) / \varepsilon$, and $\delta>0$ will be fixed later. Let us consider now equation (3.10) written for the difference of the solutions. In this case we consider $B_{N}^{-1} \tilde{\varphi}(t)$ as test function, where $\tilde{\varphi}(t)=\left(\varphi_{1}-\varphi_{2}\right)(t)-\overline{\left(\varphi_{1}-\varphi_{2}\right)} \in H_{(0)}^{1}(\Omega)$, for almost any $t \in(0, T)$. Recalling (2.5) and (3.12), we have

$$
\begin{aligned}
& \frac{1}{2} \frac{d}{d t}\left\|\varphi_{1}-\varphi_{2}\right\|_{\left(H^{1}\right)^{\prime}}^{2}=\left\langle\frac{d}{d t}\left(\varphi_{1}-\varphi_{2}\right), B_{N}^{-1} \tilde{\varphi}\right\rangle_{H^{1}} \\
& =-\left(\mu_{1}-\mu_{2}, \tilde{\varphi}\right)+\left\langle K_{2}\left(\boldsymbol{u}_{1}, \varphi_{1}\right)-K_{2}\left(\boldsymbol{u}_{2}, \varphi_{2}\right), B_{N}^{-1} \tilde{\varphi}\right\rangle_{H^{1}} \\
& =\widetilde{D}_{1}+\widetilde{D}_{2} .
\end{aligned}
$$

As far as $\widetilde{D}_{1}$ is concerned, notice that it can be written as

$$
\begin{aligned}
\widetilde{D}_{1} & =-\left(M\left(\cdot, \varphi_{1}\right)-M\left(\cdot, \varphi_{2}\right), \varphi_{1}-\varphi_{2}\right)+\left(J *\left(\varphi_{1}-\varphi_{2}\right), \varphi_{1}-\varphi_{2}\right) \\
& +\left(F^{\prime}\left(\varphi_{1}\right)-F^{\prime}\left(\varphi_{2}\right), \overline{\varphi_{1}-\varphi_{2}}\right),
\end{aligned}
$$


where we have set $M(x, s):=a(x) s+F^{\prime}(s)$. Observe that (2.3) implies $M_{s}(x, \varphi) \geq$ $\left(c_{5} / 2\right)|\varphi|^{2 q}+c_{5} / 2$ for all $s \in \mathbb{R}$ and for almost any $x \in \Omega$. Arguing as, e.g., in [31, Lemma 1.19, Chapter 5], we deduce that

$$
\left(M\left(\cdot, \varphi_{1}\right)-M\left(\cdot, \varphi_{2}\right), \varphi_{1}-\varphi_{2}\right) \geq c\left\|\varphi_{1}-\varphi_{2}\right\|_{L^{2 q+2}}^{2 q+2}+c\left\|\varphi_{1}-\varphi_{2}\right\|^{2} .
$$

Moreover, the third term on the right hand side of (5.21) can be estimated as

$$
\begin{aligned}
\left|\left(F^{\prime}\left(\varphi_{1}\right)-F^{\prime}\left(\varphi_{2}\right), \overline{\varphi_{1}-\varphi_{2}}\right)\right| & \leq\left(\left\|F^{\prime}\left(\varphi_{1}\right)\right\|_{L^{1}}+\left\|F^{\prime}\left(\varphi_{2}\right)\right\|_{L^{1}}\right)\left|\overline{\varphi_{1}-\varphi_{2}}\right| \\
& \leq c\left(1+\left\|F\left(\varphi_{1}\right)\right\|_{L^{1}}+\left\|F\left(\varphi_{2}\right)\right\|_{L^{1}}\right)\left|\overline{\varphi_{1}-\varphi_{2}}\right| \\
& \leq \Gamma\left|\overline{\varphi_{1}-\varphi_{2}}\right|
\end{aligned}
$$

where we have used the growth condition of $F$, which implies that $\left|F^{\prime}(s)\right| \leq c|F(s)|+c$, for all $s \in \mathbb{R}$. We shall henceforth denote by $\Gamma$ a positive constant which depends on the data, more precisely on $\left\|\boldsymbol{u}_{0 i}\right\|,\left\|\varphi_{0 i}\right\|,\left\|F\left(\varphi_{0 i}\right)\right\|_{L^{1}}, i=1,2$, and on $\|\boldsymbol{h}\|_{L^{p^{\prime}\left(0, T ; V_{p}^{\prime}\right)}}$ (cf. $(4.6)-(4.9))$.

Thus we have

$$
\widetilde{D}_{1} \leq-c\left\|\varphi_{1}-\varphi_{2}\right\|^{2}-c\left\|\varphi_{1}-\varphi_{2}\right\|_{L^{2 q+2}}^{2 q+2}+\left(J *\left(\varphi_{1}-\varphi_{2}\right), \varphi_{1}-\varphi_{2}\right)+\Gamma\left|\overline{\varphi_{1}-\varphi_{2}}\right|
$$

The third term on the right hand side of (5.22) is easily estimated as usual, on account of the smoothing effect of the convolution, namely

$$
\begin{aligned}
\left|\left(J *\left(\varphi_{1}-\varphi_{2}\right), \varphi_{1}-\varphi_{2}\right)\right| & \leq\left\|J *\left(\varphi_{1}-\varphi_{2}\right)\right\|_{H^{1}}\left\|\varphi_{1}-\varphi_{2}\right\|_{\left(H^{1}\right)^{\prime}} \\
& \leq\left(a^{*}+b\right)\left\|\varphi_{1}-\varphi_{2}\right\|\left\|\varphi_{1}-\varphi_{2}\right\|_{\left(H^{1}\right)^{\prime}} \\
& \leq \delta\left\|\varphi_{1}-\varphi_{2}\right\|^{2}+C_{\delta}\left\|\varphi_{1}-\varphi_{2}\right\|_{\left(H^{1}\right)^{\prime}}^{2}
\end{aligned}
$$

Finally, let us set

$$
\begin{aligned}
\widetilde{D}_{2}=\widetilde{D}_{2 a}+\widetilde{D}_{2 b} & =\left\langle K_{2}\left(\boldsymbol{u}_{1}, \varphi_{1}\right)-K_{2}\left(\boldsymbol{u}_{2}, \varphi_{1}\right), B_{N}^{-1} \tilde{\varphi}\right\rangle_{H^{1}} \\
& +\left\langle K_{2}\left(\boldsymbol{u}_{2}, \varphi_{1}\right)-K_{2}\left(\boldsymbol{u}_{2}, \varphi_{2}\right), B_{N}^{-1} \tilde{\varphi}\right\rangle_{H^{1}} .
\end{aligned}
$$

Then observe that

$$
\begin{aligned}
\left|\widetilde{D}_{2 a}\right| & \leq \int_{\Omega}\left|\varphi_{1}\right|\left|\boldsymbol{u}_{1}-\boldsymbol{u}_{2}\right|\left|\nabla B_{N}^{-1} \tilde{\varphi}\right| d x \leq\left\|\varphi_{1}\right\|_{L^{3}}\left\|\boldsymbol{u}_{1}-\boldsymbol{u}_{2}\right\|\|\tilde{\varphi}\| \\
& \leq \delta\left\|\varphi_{1}-\varphi_{2}\right\|^{2}+C_{\delta}\left\|\varphi_{1}\right\|_{L^{3+\varepsilon}}^{2}\left\|\boldsymbol{u}_{1}-\boldsymbol{u}_{2}\right\|^{2}+c\left|\overline{\varphi_{1}-\varphi_{2}}\right|^{2}
\end{aligned}
$$

and

$$
\begin{aligned}
\left|\widetilde{D}_{2 b}\right| & \leq \int_{\Omega}\left|\varphi_{1}-\varphi_{2}\right|\left|\boldsymbol{u}_{2}\right|\left|\nabla B_{N}^{-1} \tilde{\varphi}\right| d x \leq\left\|\varphi_{1}-\varphi_{2}\right\|\left\|\boldsymbol{u}_{2}\right\|_{L^{p^{*}}}\left\|\nabla B_{N}^{-1} \tilde{\varphi}\right\|_{L^{\rho}} \\
& \leq c\left\|\varphi_{1}-\varphi_{2}\right\|\left\|\boldsymbol{u}_{2}\right\|_{L^{p^{*}}}\left(\left\|\varphi_{1}-\varphi_{2}\right\|_{\left(H^{1}\right)^{\prime}}^{\theta}+\left|\overline{\varphi_{1}-\varphi_{2}}\right|^{\theta}\right)\left(\left\|\varphi_{1}-\varphi_{2}\right\|^{1-\theta}+\left|\overline{\varphi_{1}-\varphi_{2}}\right|^{1-\theta}\right) \\
& \leq \delta\left\|\varphi_{1}-\varphi_{2}\right\|^{2}+C_{\delta}\left\|\boldsymbol{u}_{2}\right\|_{V_{p}}^{p_{\text {uniq }}}\left\|\varphi_{1}-\varphi_{2}\right\|_{\left(H^{1}\right)^{\prime}}^{2}+C_{\delta}\left(1+\left\|\boldsymbol{u}_{2}\right\|_{V_{p}}^{p_{\text {uniq }}}\right)\left|\overline{\varphi_{1}-\varphi_{2}}\right|^{2} .
\end{aligned}
$$

Here $\theta$ and $\rho$ are as in (5.17), and we have interpolated the estimates (2.6).

We now sum (5.15), (5.20) and insert estimates (5.16), (5.18)-(5.19), (5.22)-(5.25) into the ensuing identity. Choosing $\delta>0$ small enough we are finally led to (5.14). Setting 
$Q=3+\varepsilon$, the function $m$ can be given by

$$
m(t):=\Gamma\left(1+\left\|\boldsymbol{u}_{2}(t)\right\|_{V_{p}}^{p_{\text {uniq }}}+\left\|\varphi_{1}(t)\right\|_{L^{Q}}^{P}+\left\|\varphi_{2}(t)\right\|_{L^{Q}}^{P}\right),
$$

which belongs to $L^{1}(0, T)$ thanks to assumptions (5.12)-(5.13).

\section{Proof of Theorem 2}

The proof is based on two lemmas. The first (see Lemma 5) shows a time regularity in Nikolskii space for an evolutionary Stokes-Ladyzhenskaya system with a given source. It can be seen as a generalization of the well-known fact that the $L^{p}\left(0, T ; V_{p}\right)$ norm of the solution is estimated by the $L^{p^{\prime}}\left(0, T ; V_{p}^{\prime}\right)$ norm of the right-hand side.

The second lemma (see Lemma 6) deals with the convective term $K_{0}(\boldsymbol{u})$, which is the critical one. It shows that if $\boldsymbol{u} \in N^{\sigma, p}\left(0, T ; V_{p}\right)$, then $K_{0}(\boldsymbol{u}) \in N^{\delta, p^{\prime}}\left(0, T ; V_{p}^{\prime}\right)$ for suitable $\delta=\delta(\sigma)$, provided that $p \geq 11 / 5$. This generalizes another well-known fact, namely that $K_{0}(\cdot)$ is bounded from $L^{p}\left(0, T ; V_{p}\right)$ into its dual if $p \geq 11 / 5$.

Lemma 5. Let $p \geq 11 / 5$ and suppose that $\boldsymbol{u} \in L^{p}\left(t_{0}, T ; V_{p}\right)$ satisfies $\frac{d}{d t} \boldsymbol{u}+N(\boldsymbol{u})=H(t)$, where $H \in N^{\delta, p^{\prime}}\left(t_{0}, T ; V_{p}^{\prime}\right)$ and $\delta p^{\prime} \leq 1, \delta \in(0,1)$. In addition, assume that $t_{0}$ is such that

$$
\left\|\boldsymbol{u}\left(t_{0}+h\right)-\boldsymbol{u}\left(t_{0}\right)\right\|^{2} \leq \mathrm{ch} \quad \text { for } h \in\left(0, h_{0}\right) \text { and for some } c>0 .
$$

Then $\boldsymbol{u} \in N^{\sigma, p}\left(t_{0}, T ; V_{p}\right)$, where $\sigma=\delta /(p-1)$.

Proof. Applying $\mathrm{d}^{h}$ to the equation and testing by $\mathrm{d}^{h} \boldsymbol{u}$, one obtains

$$
\frac{1}{2} \frac{d}{d t}\left\|\mathrm{~d}^{h} \boldsymbol{u}\right\|^{2}+\left\langle\mathrm{d}^{h} N(\boldsymbol{u}), \mathrm{d}^{h} \boldsymbol{u}\right\rangle_{V_{p}}=\left\langle\mathrm{d}^{h} H(t), \mathrm{d}^{h} \boldsymbol{u}\right\rangle_{V_{p}} .
$$

Here, on account of (2.1), we have

$$
\left\langle\mathrm{d}^{h} N(\boldsymbol{u}), \mathrm{d}^{h} \boldsymbol{u}\right\rangle_{V_{p}} \geq c_{2}\left(\left\|\mathrm{~d}^{h} \boldsymbol{u}\right\|_{V_{p}}^{p}+\left\|\mathrm{d}^{h} \boldsymbol{u}\right\|_{V_{2}}^{2}\right)
$$

Further, observe that

$$
\left\langle\mathrm{d}^{h} H(t), \mathrm{d}^{h} \boldsymbol{u}\right\rangle_{V_{p}} \leq\left\|\mathrm{d}^{h} H(t)\right\|_{V_{p}^{\prime}}\left\|\mathrm{d}^{h} \boldsymbol{u}\right\|_{V_{p}} \leq \frac{c_{2}}{2}\left\|\mathrm{~d}^{h} \boldsymbol{u}\right\|_{V_{p}}^{p}+C\left\|\mathrm{~d}^{h} H(t)\right\|_{V_{p}^{\prime}}^{p^{\prime}} .
$$

Thus we eventually get

$$
\sup _{t_{0} \leq t \leq T-h}\left\|\mathrm{~d}^{h} \boldsymbol{u}(t)\right\|^{2}+c \int_{t_{0}}^{T-h}\left(\left\|\mathrm{~d}^{h} \boldsymbol{u}\right\|_{V_{p}}^{p}+\left\|\mathrm{d}^{h} \boldsymbol{u}\right\|_{V_{2}}^{2}\right) d t \leq c_{1} h+C \int_{t_{0}}^{T-h}\left\|\mathrm{~d}^{h} H(t)\right\|_{V_{p}^{\prime}}^{p^{\prime}} d t .
$$

The last term is estimated by $c_{2} h^{\delta p^{\prime}}$ and therefore we get

$$
\frac{1}{h^{\delta /(p-1)}}\left\|d^{h} \boldsymbol{u}\right\|_{L^{p}\left(t_{0}, T-h ; V_{p}\right)} \leq C\left(h^{\frac{1}{p}-\frac{\delta}{p-1}}+1\right) .
$$

Taking the sup for $h>0$ the conclusion follows, since we have $\delta p^{\prime} \leq 1$.

Because the embedding theorem for Nikolskii spaces is not sharp (cf. (2.8)), we will repeatedly write $\gamma+\epsilon$ or $\gamma-\epsilon$ for some number strictly larger or smaller than $a$, respectively; the value $\epsilon>0$ will be arbitrarily small and its values can change from line to line. Hence we have $N^{s, p}(I ; X) \hookrightarrow L^{\widetilde{p}_{s}-\varepsilon}(I ; X)$, where $1 / \widetilde{p}_{s}=1 / p-s$. 
Lemma 6. Let $\boldsymbol{u} \in N^{\sigma, p}\left(t_{0}, T ; V_{p}\right) \cap L^{\infty}\left(t_{0}, T ; G\right)$ with $\sigma \in[0,1)$ and $p \geq 11 / 5$. In addition, assume that (6.1) holds. Then, we have

$$
K_{0}(\boldsymbol{u}) \in N^{\delta, p^{\prime}}\left(t_{0}, T ; V_{p}^{\prime}\right)
$$

where

$$
\delta=\left\{\begin{array}{l}
\frac{5 p-9}{2(5 p-6)}+\frac{3 \sigma}{5 p-6}, \quad \text { if } p \geq \frac{13}{5} \text { or if } p<\frac{13}{5} \text { and } \sigma_{p}<\sigma<1 \\
\frac{5 p-11}{5 p-6}+\frac{6 \sigma}{5 p-6}, \quad \text { if } p<\frac{13}{5} \text { and } 0 \leq \sigma \leq \sigma_{p} .
\end{array}\right.
$$

with $\sigma_{p}:=\frac{13-5 p}{6}$.

Proof. Let $\mathbf{v} \in L^{p}\left(t_{0}, T-h ; V_{p}\right)$ with $\|\mathbf{v}\|_{L^{p}\left(t_{0} ; T-h ; V_{p}\right)} \leq 1$. Observe that

$$
\begin{aligned}
& \left|\left\langle\mathrm{d}^{h} K_{0}(\boldsymbol{u}), \mathbf{v}\right\rangle_{L^{p}\left(t_{0} ; T-h ; V_{p}\right)}\right| \\
& \leq \int_{\Omega \times\left(t_{0}, T-h\right)}\left|\mathrm{d}^{h} \boldsymbol{u}\right|\left(|\boldsymbol{u}|+\left|\tau^{h} \boldsymbol{u}\right|\right)|\nabla \mathbf{v}| d x d t \\
& \leq \int_{t_{0}}^{T-h}\left\|\mathrm{~d}^{h} \boldsymbol{u}\right\|_{L^{2 p^{\prime}}}\left(\|\boldsymbol{u}\|_{L^{2 p^{\prime}}}+\left\|\tau^{h} \boldsymbol{u}\right\|_{L^{2 p^{\prime}}}\right)\|\nabla \mathbf{v}\|_{L^{p}} d t \\
& \leq C \int_{t_{0}}^{T-h}\left\|\mathrm{~d}^{h} \boldsymbol{u}\right\|^{1-\gamma}\left\|\mathrm{d}^{h} \boldsymbol{u}\right\|_{V_{p}}^{\gamma}\left(\|\boldsymbol{u}\|_{V_{p}}^{\gamma}+\left\|\tau^{h} \boldsymbol{u}\right\|_{V_{p}}^{\gamma}\right)\|\mathbf{v}\|_{V_{p}} d t .
\end{aligned}
$$

In the last inequality we have used the interpolation (5.4), as well as the regularity $\boldsymbol{u} \in L^{\infty}\left(t_{0}, T ; G\right)$ and the embedding $V_{p} \hookrightarrow L^{p^{*}}(\Omega)$.

Let us first consider the case $\sigma \in(0,1)$. In this case we estimate the last term of $(6.4)$ by means of Hölder's inequality with exponents $r_{\sigma}+\varepsilon^{\prime}, p / \gamma,\left(\widetilde{p}_{\sigma}-\varepsilon\right) / \gamma$ and $p$, where $\widetilde{p}_{\sigma}$ is given by (2.8), $r_{\sigma}$ is computed by Hölder's condition, namely

$$
\frac{1}{r_{\sigma}}=\frac{5 p-11}{5 p-6}+\sigma \gamma
$$

and $\varepsilon, \varepsilon^{\prime}>0$ are such that $1 /\left(r_{\sigma}+\varepsilon^{\prime}\right)+\gamma / p+\gamma /\left(\widetilde{p}_{\sigma}-\varepsilon\right)+1 / p=1$. Therefore, we get

$$
\begin{aligned}
\left|\left\langle\mathrm{d}^{h} K_{0}(\boldsymbol{u}), \mathbf{v}\right\rangle_{L^{p}\left(t_{0} ; T-h ; V_{p}\right)}\right| & \leq C\left\|\mathrm{~d}^{h} \boldsymbol{u}\right\|_{L^{(1-\gamma)\left(r_{\sigma}+\varepsilon^{\prime}\right)}\left(t_{0}, T-h ; G\right)}^{1-\gamma}\left\|\mathrm{d}^{h} \boldsymbol{u}\right\|_{L^{p}\left(t_{0}, T-h ; V_{p}\right)}^{\gamma} \\
& \cdot\left(\|\boldsymbol{u}\|_{L^{\tilde{p}_{\sigma}-\varepsilon}\left(t_{0}, T ; V_{p}\right)}^{\gamma}+\left\|\tau^{h} \boldsymbol{u}\right\|_{L^{\tilde{p}_{\sigma}-\varepsilon}\left(t_{0}, T-h ; V_{p}\right)}^{\gamma}\right) .
\end{aligned}
$$

Let us begin to estimate the first factor on the right hand side of the first inequality in (6.5). Observe first that, on account of (3.5), (4.41) and employing Lemma 1 with $X=V_{p}, H=G, \alpha=0$ and $\beta=1$, we obtain $\boldsymbol{u} \in N^{1 / 2,2}(0, T ; G)$, which implies that

$$
\left\|\mathrm{d}^{h} \boldsymbol{u}\right\|_{L^{2}\left(t_{0}, T-h ; G\right)} \leq c h^{1 / 2} .
$$

Next, we observe that we have

$$
(1-\gamma) r_{\sigma}>2 \Longleftrightarrow \frac{2(5 p-11)}{5 p-9}+\frac{6 \sigma}{5 p-9}<1 .
$$

Therefore, since $2(5 p-11) /(5 p-9)>1$ iff $p>13 / 5$ (recall that $p \geq 11 / 5)$, then we have $(1-\gamma) r_{\sigma}<2$ for $p \geq 13 / 5$ and for all $\sigma \in(0,1)$ (recall that we are considering the case $\sigma \in(0,1))$. In this case, taking $\varepsilon$ (and hence $\varepsilon^{\prime}$ ) small enough, by (6.6) we have

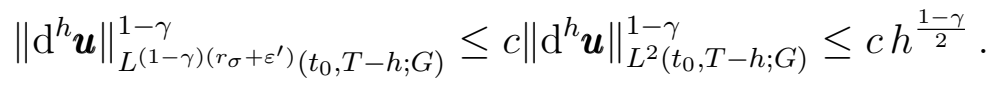


On the other hand, if $11 / 5 \leq p<13 / 5$ and $\sigma \in(0,1)$ is such that $(1-\gamma) r_{\sigma} \geq 2$, then, using interpolation, the fact that $\boldsymbol{u} \in L^{\infty}(0, T ; G)$, and (6.6) again, we have

$$
\begin{aligned}
& \left\|\mathrm{d}^{h} \boldsymbol{u}\right\|_{L^{(1-\gamma)\left(r_{\sigma}+\varepsilon^{\prime}\right)}\left(t_{0}, T-h ; G\right)}^{1-\gamma} \leq\left\|\mathrm{d}^{h} \boldsymbol{u}\right\|_{L^{\infty}\left(t_{0}, T-h ; G\right)}^{1-\gamma-\frac{2}{r_{\sigma}+\varepsilon^{\prime}}}\left\|\mathrm{d}^{h} \boldsymbol{u}\right\|_{L^{2}\left(t_{0}, T-h ; G\right)}^{\frac{2}{r_{\sigma}+\epsilon^{\prime}}} \\
& \leq C\left\|\mathrm{~d}^{h} \boldsymbol{u}\right\|_{L^{2}\left(t_{0}, T-h ; G\right)}^{\frac{2}{r_{\sigma}}} \leq C h^{\frac{1}{r_{\sigma}}} .
\end{aligned}
$$

We can easily check that the condition $(1-\gamma) r_{\sigma} \geq 2$ is satisfied if and only if we have

$$
\frac{2(5 p-11)}{5 p-9}+\frac{6 \sigma}{5 p-9} \leq 1
$$

namely, if and only if $\sigma \leq \sigma_{p}$, where $\sigma_{p}:=(13-5 p) / 6$.

Moreover, if $11 / 5 \leq p<13 / 5$ and $\sigma \in(0,1)$ is such that $(1-\gamma) r_{\sigma}<2$ (namely $\left.\sigma_{p}<\sigma<1\right)$, then we argue again as in (6.8).

The second and the third factors on the right hand side of the first inequality in (6.5) are estimated by using, respectively, the fact that $\boldsymbol{u} \in N^{\sigma, p}\left(t_{0}, T ; V_{p}\right)$, which implies that $\left\|\mathrm{d}^{h} \boldsymbol{u}\right\|_{L^{p}\left(t_{0}, T-h ; V_{p}\right)} \leq C h^{\sigma}$ for all $h>0$, and the embedding $N^{\sigma, p}\left(t_{0}, T ; V_{p}\right) \hookrightarrow$ $L^{\widetilde{p}_{\sigma}-\varepsilon}\left(t_{0}, T ; V_{p}\right)$ (cf. (2.8)). Let us now take the sup in (6.5) over all $\mathbf{v} \in L^{p}\left(t_{0}, T-h ; V_{p}\right)$ with $\|\mathbf{v}\|_{L^{p}\left(t_{0} ; T-h ; V_{p}\right)} \leq 1$ and use (6.8)-(6.9). In the case $p \geq 13 / 5$ and all $\sigma \in(0,1)$ or in the case $11 / 5 \leq p<13 / 5$ and $\sigma \in(0,1)$ such that $(1-\gamma) r_{\sigma}<2$, we get

$$
\left\|\mathrm{d}^{h} K_{0}(\boldsymbol{u})\right\|_{L^{p^{\prime}}\left(t_{0}, T-h ; V_{p}^{\prime}\right)} \leq C h^{\frac{1-\gamma}{2}+\sigma \gamma},
$$

which implies that $K_{0}(\boldsymbol{u}) \in N^{\delta, p^{\prime}}\left(t_{0}, T ; V_{p}^{\prime}\right)$, with $\delta:=(1-\gamma) / 2+\sigma \gamma$. In the case $11 / 5 \leq p<13 / 5$ and $\sigma \in(0,1)$ such that $(1-\gamma) r_{\sigma} \geq 2$ we get

$$
\left\|\mathrm{d}^{h} K_{0}(\boldsymbol{u})\right\|_{L^{p^{\prime}\left(t_{0}, T-h ; V_{p}^{\prime}\right)}} \leq C h^{\frac{1}{r_{\sigma}}+\sigma \gamma}
$$

which implies that $K_{0}(\boldsymbol{u}) \in N^{\delta, p^{\prime}}\left(t_{0}, T ; V_{p}^{\prime}\right)$, with $\delta:=1 / r_{\sigma}+\sigma \gamma$.

Let us now consider the case $\sigma=0$ (this case is crucial, since it will be the starting point of the iteration procedure in the proof of Theorem 2). The last term in (6.4) has to be estimated a bit differently. However, we can see that (6.3) still holds (hence, we have $\delta=(5 p-9) / 2(5 p-6))$. Indeed, we now use Hölder's inequality with exponents $r_{0}=(5 p-6) /(5 p-11), p / \gamma, p / \gamma$, and $p$. Notice that $p=11 / 5$ is allowed (in this case $\left.r_{0}=\infty\right)$. Hence, instead of (6.5), we simply write

$$
\left|\left\langle\mathrm{d}^{h} K_{0}(\boldsymbol{u}), \mathbf{v}\right\rangle_{L^{p}\left(t_{0} ; T-h ; V_{p}\right)}\right| \leq C\left\|\mathrm{~d}^{h} \boldsymbol{u}\right\|_{L^{(1-\gamma) r_{0}\left(t_{0}, T-h ; G\right)}}^{1-\gamma}\|\boldsymbol{u}\|_{L^{p}\left(t_{0}, T ; V_{p}\right)}^{2 \gamma},
$$

and then we argue as above, by using, respectively, (6.8) and (6.9).

Proof of Theorem 2. If $p \geq 5 / 2$, then $p_{\text {uniq }} \leq p$ and there is nothing to prove; hence we will assume that $11 / 5<p<5 / 2$. By the embedding properties of Nikolskii spaces, it is enough to establish that $\boldsymbol{u} \in N^{\sigma_{\text {uniq }}+\epsilon, p}\left(t_{0}, T ; V_{p}\right)$, where $\sigma_{\text {uniq }}=5 / 2 p-1$. By Lemma 3 , we can assume that (6.1) holds true. Thus, in virtue of (3.8) and of Lemma 5 , it will be enough to prove that

$$
K_{0}(\boldsymbol{u})+K_{1}(\varphi)+\boldsymbol{h} \in N^{\delta_{\mathrm{uniq}}+\epsilon, p^{\prime}}\left(t_{0}, T ; V_{p}^{\prime}\right) \quad \delta_{\text {uniq }}=(p-1)\left(\frac{5}{2 p}-1\right) .
$$

For $\boldsymbol{h}$ this is just our assumption. Concerning $K_{1}(\varphi)$, we have

$$
\mathrm{d}^{h} K_{1}(\varphi)=-\frac{1}{2} \mathrm{~d}^{h} \varphi^{2} \nabla a+\left(\nabla J * \mathrm{~d}^{h} \varphi\right) \varphi+\tau^{h}(\nabla J * \varphi) \mathrm{d}^{h} \varphi
$$


We will only estimate the second term, others being similar or even simpler. For $\mathbf{v} \in$ $L^{p}\left(t_{0}, T-h ; V_{p}\right)$, with $\|\mathbf{v}\|_{L^{p}\left(t_{0} ; T-h ; V_{p}\right)} \leq 1$, we write

$$
\begin{aligned}
\left|\left\langle\left(\nabla J * \mathrm{~d}^{h} \varphi\right) \varphi, \mathbf{v}\right\rangle_{L^{p}\left(t_{0}, T-h ; V_{p}\right)}\right| & \leq \int_{\Omega \times\left(t_{0}, T-h\right)}\left|\nabla J * \mathrm{~d}^{h} \varphi\|\varphi\| \mathbf{v}\right| \\
& \leq b \int_{t_{0}}^{T-h}\left\|\mathrm{~d}^{h} \varphi\right\|\|\varphi\|_{L^{2 q+2}}\|\mathbf{v}\|_{\left(L^{2(1+1 / q)}\right)^{3}} d t \\
& \leq C \int_{t_{0}}^{T-h}\left\|\mathrm{~d}^{h} \varphi\right\|\|\mathbf{v}\|_{V_{p}} d t \\
& \leq C\left\|\mathrm{~d}^{h} \varphi\right\|_{L^{2}\left(t_{0}, T-h ; L^{2}(\Omega)\right)} \leq C h^{1 / 2} .
\end{aligned}
$$

We have used (3.9) and the fact that we have $V_{p} \hookrightarrow L^{p^{*}}(\Omega)^{3} \hookrightarrow L^{2(1+1 / q)}(\Omega)^{3}$, where the last embedding holds thanks to the condition $q \geq q_{p}$ (cf. (4.18)). Moreover, the last estimate in (6.11) is due to the fact that $\varphi \in N^{1 / 2,2}\left(0, T ; L^{2}(\Omega)\right)$ as follows from Lemma 1 and (3.6), (4.25). Estimate (6.11) implies that

$$
\left\|\left(\nabla J * \mathrm{~d}^{h} \varphi\right) \varphi\right\|_{L^{p^{\prime}}\left(t_{0}, T-h ; V_{p}^{\prime}\right)} \leq C h^{1 / 2},
$$

and hence we get $K_{1}(\varphi) \in N^{1 / 2, p^{\prime}}\left(t_{0}, T ; V_{p}^{\prime}\right)$. Observe that $\delta_{\text {uniq }}<1 / 2$. It remains to consider the convective term $K_{0}(\boldsymbol{u})$, which is the most delicate.

For this term we can employ an iterative scheme. More precisely, if $\boldsymbol{u} \in N^{\sigma, p}\left(t_{0}, T ; V_{p}\right)$, it follows from Lemmas 5 and 6 that $\boldsymbol{u} \in N^{\tilde{\sigma}, p}\left(t_{0}, T ; V_{p}\right)$, where

$$
\tilde{\sigma}=\Psi(\sigma) \quad \Psi(\sigma):= \begin{cases}\frac{5 p-11}{(5 p-6)(p-1)}+\frac{6 \sigma}{(5 p-6)(p-1)}, & \text { if } 0 \leq \sigma \leq \sigma_{p} \\ \frac{5 p-9}{2(5 p-6)(p-1)}+\frac{3 \sigma}{(5 p-6)(p-1)}, & \text { if } \quad \sigma_{p}<\sigma<1 .\end{cases}
$$

Hence, starting from $\sigma=0$, on account of the fact that $p>11 / 5$, we can arrive arbitrarily close to the fixed point $\sigma_{\max }$ which is the solution of

$$
\frac{5 p-9}{2(5 p-6)(p-1)}+\frac{3 \sigma}{(5 p-6)(p-1)}=\sigma
$$

namely

$$
\sigma_{\max }=\frac{5 p-9}{2\left(5 p^{2}-11 p+3\right)} .
$$

We can now see that, if $11 / 5<p<5 / 2$, then we have $\sigma_{\max }>\sigma_{\text {uniq. }}$. Indeed, we have $\sigma_{\max }>(5 p-9) / 2 p(5 p-8)>\sigma_{\text {uniq }}=(5-2 p) / 2 p$, since $10 p^{2}-36 p+31>0$ when $p>11 / 5>(18+\sqrt{14}) / 10$.

Moreover, we can check that the condition $\delta p^{\prime} \leq 1$ of Lemma 5 is satisfied at every step of the iteration. Let us check it, e.g., for $\sigma_{p}<\sigma<\sigma_{\max }$. For these values of $\sigma$ we have

$$
\begin{aligned}
\delta p^{\prime} & =\left(\frac{5 p-9}{2(5 p-6)}+\frac{3 \sigma}{(5 p-6)}\right) \frac{p}{p-1} \leq\left(\frac{5 p-9}{2(5 p-6)}+\frac{3 \sigma_{\max }}{(5 p-6)}\right) \frac{p}{p-1}=p \sigma_{\max } \\
& =\frac{(5 p-9) p}{2\left(5 p^{2}-11 p+3\right)}<\frac{(5 p-9) p}{2\left(5 p^{2}-11 p+p\right)}=\frac{(5 p-9)}{2(5 p-10)}<1 .
\end{aligned}
$$

In the last two inequalities we have used the fact that $p<5 / 2$ and $p>11 / 5$, respectively. Therefore, the proof is concluded after finitely many steps.

To prove the second part of the theorem, namely that $\boldsymbol{u} \in L_{l o c}^{p_{\text {uniq }}}\left(0, T ; V_{p}\right)$ for any weak solution, it is enough to note that almost every $t_{0} \in(0, T)$ satisfies the assumption of the 
initial regularity: $\boldsymbol{u}\left(t_{0}\right) \in V_{p}$, and $t=t_{0}$ is a Lebesgue point of the (integrable) function $t \mapsto\|\boldsymbol{h}(t)\|_{V_{p}^{\prime}}^{p^{\prime}}$

Proof of Corollary 1. Let the weak solutions $\left[\boldsymbol{u}_{1}, \varphi_{1}\right],\left[\boldsymbol{u}_{2}, \varphi_{2}\right]$ coincide on $\left[t_{0}, t_{0}+\tau\right]$, where $\tau \in\left(0, T-t_{0}\right]$. Take $\tilde{t}_{0} \in\left[t_{0}, t_{0}+\tau\right]$ such that $\boldsymbol{u}_{1}\left(\tilde{t}_{0}\right) \in V_{p}$, and $t=\tilde{t}_{0}$ is a Lebesgue point of $t \mapsto\|\boldsymbol{h}(t)\|_{V_{p}^{\prime}}^{p^{\prime}}$. By Theorem 2, solution $\boldsymbol{u}_{1}$ has the regularity $\boldsymbol{u}_{1} \in L^{p_{\text {uniq }}}\left(\widetilde{t}_{0}, T ; V_{p}\right)$. Moreover, the regularity (5.13) is provided by (3.6) and by the condition $q>1 / 2$. By applying Lemma 4 and Gronwall's lemma we therefore deduce that $\left[\boldsymbol{u}_{1}, \varphi_{1}\right],\left[\boldsymbol{u}_{2}, \varphi_{2}\right]$ coincide on $\left[\widetilde{t}_{0}, T\right]$, and hence also on $\left[t_{0}, T\right]$ (on $\left[t_{0}, \widetilde{t}_{0}\right]$ they coincide by assumption).

Proof of Corollary 2. By the above we know that $\boldsymbol{u} \in L^{p_{\text {uniq }}}\left(t_{0}, T ; V_{p}\right)$. We apply Lemma 4 with $\boldsymbol{u}_{1}=\tau^{h} \boldsymbol{u}, \boldsymbol{u}_{2}=\boldsymbol{u}, \varphi_{1}=\tau^{h} \varphi$ and $\varphi_{2}=\varphi$. There appears an additional term on the right-hand side due to the time difference of the external force $\boldsymbol{h}$, which is estimated as follows:

$$
\left|\left\langle\mathrm{d}^{h} \boldsymbol{h}(t), \mathrm{d}^{h} \boldsymbol{u}\right\rangle\right| \leq\left\|\mathrm{d}^{h} \boldsymbol{h}(t)\right\|_{V_{p}^{\prime}}\|\boldsymbol{u}\|_{V_{p}} \leq \epsilon\left\|\mathrm{d}^{h} \boldsymbol{u}\right\|_{V_{p}}^{p}+C_{\epsilon}\|\boldsymbol{h}(t)\|_{V_{p}^{\prime}}^{p^{\prime}}
$$

and the first term is absorbed in the left-hand side. We can now apply Gronwall's lemma. Note that $\overline{\varphi_{1}-\varphi_{2}}=0$. The difference of initial conditions is estimated by $c h$ (see (5.6)). Finally, the integral term with $\|\boldsymbol{h}\|_{V_{p}^{\prime}}^{p^{\prime}}$ is controlled by $c h^{\delta}$, from which the conclusion follows immediately, but for the $L^{\infty}(\Omega \times(0, T))$ bound on $\varphi$.

This is proved from the fact that $\varphi\left(t_{0}\right) \in L^{\infty}(\Omega)$, by means of the so-called Alikakos's iteration argument (see, e.g., [5, Theorem 2.1]). The technique employs $|\varphi|^{r-2} \varphi$ as a test function in (1.15), with $r$ arbitrarily large. This is not possible in the class of weak solutions in general. However, the weak solutions are unique in the given situation. Hence they can be obtained as limit of smooth solutions of a suitably formulated approximating problem, for which the $L^{\infty}$ bound can be proved rigorously. This bound is then preserved by the lower-semicontinuity of $L^{\infty}$ norm in the weak ${ }^{*}$-limit (cf., for instance, the truncation argument used in [18, Proof of Theorem 3]).

Acknowledgments. The first two authors are members of the Gruppo Nazionale per l'Analisi Matematica, la Probabilità e le loro Applicazioni (GNAMPA) of the Istituto Nazionale di Alta Matematica (INdAM). S.F. is "titolare di un Assegno di Ricerca dell'Istituto Nazionale di Alta Matematica".

\section{REFERENCES}

[1] H. Abels. On a diffuse interface model for two-phase flows of viscous, incompressible fluids with matched densities. Arch. Ration. Mech. Anal., 194(2):463-506, 2009.

[2] H. Abels, L. Diening, and Y. Terasawa. Existence of weak solutions for a diffuse interface model of non-Newtonian two-phase flows. Nonlinear Anal. Real World Appl., 15:149-157, 2014.

[3] R. A. Adams and J. J. F. Fournier. Sobolev spaces, volume 140 of Pure and Applied Mathematics (Amsterdam). Elsevier/Academic Press, Amsterdam, second edition, 2003.

[4] D. M. Anderson, G. B. McFadden, and A. A. Wheeler. Diffuse-interface methods in fluid mechanics. volume 30 of Annu. Rev. Fluid Mech., pages 139-165. Annual Reviews, Palo Alto, CA, 1998.

[5] P. W. Bates and J. Han. The Neumann boundary problem for a nonlocal Cahn-Hilliard equation. J. Differential Equations, 212(2):235-277, 2005.

[6] C. Bennett and R. Sharpley. Interpolation of operators, volume 129 of Pure and Applied Mathematics. Academic Press, Inc., Boston, MA, 1988. 
[7] S. Bosia. Analysis of a Cahn-Hilliard-Ladyzhenskaya system with singular potential. J. Math. Anal. Appl., 397(1):307-321, 2013.

[8] S. Bosia and S. Gatti. Pullback exponential attractor for a Cahn-Hilliard-Navier-Stokes system in 2D. Dyn. Partial Differ. Equ., 11(1):1-38, 2014.

[9] F. Boyer. Mathematical study of multi-phase flow under shear through order parameter formulation. Asymptot. Anal., 20(2):175-212, 1999.

[10] M. Bulíček, F. Ettwein, P. Kaplický, and D. Pražák. On uniqueness and time regularity of flows of power-law like non-Newtonian fluids. Math. Methods Appl. Sci., 33(16):1995-2010, 2010.

[11] P. Colli, S. Frigeri, and M. Grasselli. Global existence of weak solutions to a nonlocal Cahn-HilliardNavier-Stokes system. J. Math. Anal. Appl., 386(1):428-444, 2012.

[12] P. Colli, P. Krejčí, E. Rocca, and J. Sprekels. Nonlinear evolution inclusions arising from phase change models. Czechoslovak Math. J., 57(132)(4):1067-1098, 2007.

[13] L. Diening, M. Růžička, and J. Wolf. Existence of weak solutions for unsteady motions of generalized Newtonian fluids. Ann. Sc. Norm. Super. Pisa Cl. Sci. (5), 9(1):1-46, 2010.

[14] S. Frigeri, C. G. Gal, and M. Grasselli. On nonlocal Cahn-Hilliard-Navier-Stokes systems in two dimensions. J. Nonlinear Sci., 26(4):847-893, 2016.

[15] S. Frigeri and M. Grasselli. Global and trajectory attractors for a nonlocal Cahn-Hilliard-NavierStokes system. J. Dynam. Differential Equations, 24(4):827-856, 2012.

[16] S. Frigeri and M. Grasselli. Nonlocal Cahn-Hilliard-Navier-Stokes systems with singular potentials. Dyn. Partial Differ. Equ., 9(4):273-304, 2012.

[17] S. Frigeri, M. Grasselli, and P. Krejčí. Strong solutions for two-dimensional nonlocal Cahn-HilliardNavier-Stokes systems. J. Differential Equations, 255(9):2587-2614, 2013.

[18] S. Frigeri, M. Grasselli, and E. Rocca. A diffuse interface model for two-phase incompressible flows with non-local interactions and non-constant mobility. Nonlinearity, 28(5):1257-1293, 2015.

[19] C. G. Gal, A. Giorgini, and M. Grasselli. The nonlocal Cahn-Hilliard equation with singular potential: well-posedness, regularity and strict separation property. J. Differential Equations, in press.

[20] C. G. Gal and M. Grasselli. Asymptotic behavior of a Cahn-Hilliard-Navier-Stokes system in 2D. Ann. Inst. H. Poincaré Anal. Non Linéaire, 27(1):401-436, 2010.

[21] G. Giacomin and J. L. Lebowitz. Phase segregation dynamics in particle systems with long range interactions. I. Macroscopic limits. J. Statist. Phys., 87(1-2):37-61, 1997.

[22] G. Giacomin and J. L. Lebowitz. Phase segregation dynamics in particle systems with long range interactions. II. Interface motion. SIAM J. Appl. Math., 58(6):1707-1729, 1998.

[23] M. Grasselli and D. Pražák. Longtime behavior of a diffuse interface model for binary fluid mixtures with shear dependent viscosity. Interfaces Free Bound., 13(4):507-530, 2011.

[24] M. Grasselli and D. Pražák. Regularity results for a Cahn-Hilliard-Navier-Stokes system with shear dependent viscosity. Z. Anal. Anwend., 33(3):271-288, 2014.

[25] M. E. Gurtin, D. Polignone, and J. Viñals. Two-phase binary fluids and immiscible fluids described by an order parameter. Math. Models Methods Appl. Sci., 6(6):815-831, 1996.

[26] M. Heida, J. Málek, and K. R. Rajagopal. On the development and generalizations of Cahn-Hilliard equations within a thermodynamic framework. Z. Angew. Math. Phys., 63(1):145-169, 2012.

[27] N. Kim, L. Consiglieri, and J. F. Rodrigues. On non-Newtonian incompressible fluids with phase transitions. Math. Methods Appl. Sci., 29(13):1523-1541, 2006.

[28] O. A. Ladyzhenskaya. New equations for the description of motion of viscous incompressible fluids and solvability in the large of boundary value problems for them. Tr. Mat. Inst. Steklova, 102:85-104, 1967.

[29] J.-L. Lions. Quelques méthodes de résolution des problèmes aux limites non linéaires. Dunod, 1969.

[30] C. Liu and J. Shen. A phase field model for the mixture of two incompressible fluids and its approximation by a Fourier-spectral method. Phys. D, 179(3-4):211-228, 2003.

[31] J. Málek, J. Nečas, M. Rokyta, and M. Růžička. Weak and measure-valued solutions to evolutionary PDEs, volume 13 of Applied Mathematics and Mathematical Computation. Chapman \& Hall, London, 1996.

[32] J. Málek and K. R. Rajagopal. Mathematical issues concerning the Navier-Stokes equations and some of its generalizations. In Evolutionary equations. Vol. II, Handb. Differ. Equ., pages 371-459. Elsevier/North-Holland, Amsterdam, 2005. 
[33] J. Simon. Sobolev, Besov and Nikol'skiur fractional spaces: imbeddings and comparisons for vector valued spaces on an interval. Ann. Mat. Pura Appl. (4), 157:117-148, 1990.

[34] V. N. Starovoitov. The dynamics of a two-component fluid in the presence of capillary forces. Math. Notes, 62(2):244-254, 1997.

[35] R. Temam. Navier-Stokes equations. AMS Chelsea Publishing, Providence, RI, 2001. Theory and numerical analysis, Reprint of the 1984 edition.

[36] L. Zhao, H. Wu, and H. Huang. Convergence to equilibrium for a phase-field model for the mixture of two viscous incompressible fluids. Commun. Math. Sci., 7(4):939-962, 2009.

* Dipartimento di Matematica e Fisica "N. Tartaglia"

Università Cattolica del Sacro Cuore (Brescia)

Via Musei 41

25121, BREscia, ItALY

E-mail address: sergiopietro.frigeri@unicatt.it, sergio.frigeri.sf@gmail.com

†Dipartimento di Matematica

Politecnico di Milano

VIA E. BONARDI 9

I-20133 Milano, ItAly

E-mail address: maurizio.grasselli@polimi.it

†DEPARTMENT OF MATHEMATICAL ANALYSis

Charles University

SOKOLOVSKÁ 83

CZ-18675 Prague 8, Czech Republic

E-mail address: prazak@karlin.mff.cuni.cz 